\title{
Evaluation of lodine-123 and lodine-131 SPECT Activity Quantification: A Monte Carlo Study
}

\section{Michaella Morphis ( $\nabla$ michaellamorphis@icloud.com )}

University of the Free State Faculty of Health Sciences https://orcid.org/0000-0002-3100-1618

Johan A. van Staden

University of the Free State Faculty of Health Sciences

Hanlie du Raan

University of the Free State Faculty of Health Sciences

Michael Ljungberg

Lund University: Lunds Universitet

Original research

Keywords: Monte Carlo simulations, SIMIND, quantification accuracy, 123I, 131I, SPECT

Posted Date: June 1st, 2021

DOI: https://doi.org/10.21203/rs.3.rs-549958/v1

License: (c) (1) This work is licensed under a Creative Commons Attribution 4.0 International License.

Read Full License

Version of Record: A version of this preprint was published at EJNMMI Physics on August 19th, 2021. See the published version at https://doi.org/10.1186/s40658-021-00407-1. 
Evaluation of Iodine-123 and Iodine-131 SPECT activity quantification: A Monte Carlo study

Michaella Morphis ${ }^{\mathrm{a}, *}$

a Department of Medical Physics, Faculty of Health Sciences, University of the Free State, Bloemfontein, South Africa

Email address: michaellamorphis@icloud.com

Johan A. van Staden ${ }^{a}$

a Department of Medical Physics, Faculty of Health Sciences, University of the Free State, Bloemfontein, South Africa

Email address: Gnbijvs@ufs.ac.za

\section{Hanlie du Raan ${ }^{\mathrm{a}}$}

a Department of Medical Physics, Faculty of Health Sciences, University of the Free State, Bloemfontein, South Africa

Email address: DuRaanH@ufs.ac.za

Michael Ljungberg ${ }^{b}$

${ }^{\mathrm{b}}$ Medical Radiation Physics, Lund University, Lund, Sweden

Email address: Michael.ljungberg@med.lu.se

\section{*Corresponding author}

Ms M. Morphis

Medical Physics (G68), Faculty of Health Sciences

University of the Free State, PO Box 339, Bloemfontein, 9300

South Africa

Telephone: +27 (0)51 4053156

Cell phone: +27 (0)72 4801722

Email address: michaellamorphis@icloud.com 


\begin{abstract}
Purpose: The quantitative accuracy of Nuclear Medicine images, acquired for both planar and SPECT studies, is influenced by the isotope-collimator combination as well as image corrections incorporated in the iterative reconstruction process. These factors can be investigated and optimised using Monte Carlo simulations. This study aimed to evaluate SPECT quantification accuracy for ${ }^{123}$ I with both the low energy high resolution (LEHR) and medium energy (ME) collimators, and ${ }^{131}$ I with the high energy (HE) collimator.

Methods: Simulated SPECT projection images were reconstructed using the OS-EM iterative algorithm, which was optimised for the number of updates, with appropriate corrections for scatter, attenuation, and collimator detector response (CDR), including septal scatter and penetration compensation. An appropriate conversion factor $(\mathrm{CF})$ was determined from four different source geometries (activity-filled: water-filled cylindrical phantom, sphere in waterfilled (cold) cylindrical phantom, sphere in air and point-like source), investigated with different VOI diameters. Recovery curves were constructed from recovery coefficients to correct for partial volume effects (PVEs). The quantitative method was evaluated for spheres in voxel-based digital cylindrical and patient phantoms.
\end{abstract}

Results: The optimal number of OS-EM updates was 60 for all isotope-collimator combinations. The $\mathrm{CF}_{\text {point }}$ with a VOI diameter equal to the physical size plus a $3.0 \mathrm{~cm}$ margin was selected, for all isotope-collimator geometries. The spheres' quantification errors in the voxel-based digital cylindrical and patient phantoms were less than $3.2 \%$ and $5.4 \%$, respectively, for all isotope-collimator combinations.

Conclusion: The study showed that quantification errors of less than $6.0 \%$ could be attained, for all isotope-collimator combinations, if corrections for; scatter, attenuation, CDR (including septal scatter and penetration) and PVEs, are performed. ${ }^{123}$ I LEHR and ${ }^{123}$ I ME quantification accuracies compared well when appropriate corrections for septal scatter and penetration were applied. This can be useful in departments that perform ${ }^{123}$ I studies and may not have access to ME collimators.

Keywords: Monte Carlo simulations, SIMIND, quantification accuracy, ${ }^{123} \mathrm{I},{ }^{131} \mathrm{I}$, SPECT 


\section{Introduction}

The branch theragnostics (1) has become a milestone in personalised cancer treatment, bridging the gap between Oncotherapy and Nuclear Medicine (NM). The principle of theragnostics lies in the combination of individualised targeted imaging of a cancer disease and its therapy. Both the diagnostic imaging and therapy procedures typically use the same pharmaceutical; however, in some cases, the diagnostic isotope may differ from that used in therapy. This allows for the visualisation of potential target volumes, such as tumours and organs at risk, enabling estimation of potential toxicities and predicting the benefits of such therapy $(2,3)$. Metaiodobenzylguanidine (mIBG), a noradrenaline analogue, was developed in the early 1970s to image tumours in the adrenal medulla (4). NM imaging using mIBG labelled to iodine-123 $\left({ }^{123} \mathrm{I}\right)$ or iodine-131 $\left({ }^{131} \mathrm{I}\right)$ has long employed the theragnostics concept. However, due to the recent development of peptide agents, the theragnostics approach has been heightened and the importance of accurate image quantification using ${ }^{123} \mathrm{I}$ and ${ }^{131} \mathrm{I}$ re-emphasised $(2,5,6)$.

In the ${ }^{123}$ I decay, mainly photons are emitted $(159.0 \mathrm{keV}$ with $83 \%$ abundance, $27.3 \mathrm{keV}$ with $71 \%$ abundance and $528.9 \mathrm{keV}$ with $1 \%$ abundance) and the decay has a physical half-life of 13.2 hours. ${ }^{131} \mathrm{I}$, with a physical half-life of 8.04 days, emits both photons (364.5 keV with $82 \%$ abundance, $636.9 \mathrm{keV}$ with $7 \%$ abundance and $722.9 \mathrm{keV}$ with $1.8 \%$ abundance) and several beta particles (with a maximum energy of $606.0 \mathrm{keV}$ and mean energy of $192.0 \mathrm{keV}$ ). Due to its shorter half-life and absence of beta particles, ${ }^{123} \mathrm{I}$ carries a reduced radiation burden compared to ${ }^{131} \mathrm{I}$, allowing for a higher administered activity amount for diagnostic imaging and is therefore better suited and favoured for diagnostic imaging. However, in centres where ${ }^{123}$ I is not available, ${ }^{131} \mathrm{I}$ may be administered in lower activity quantities for diagnostic imaging $(7,8)$. Both ${ }^{123} \mathrm{I}-\mathrm{mIBG}$ and ${ }^{131} \mathrm{I}-\mathrm{mIBG}$ accumulate in neuroendocrine tumours (NETs) as well as in the lungs, liver, kidneys, spleen, bladder, bone marrow and salivary glands $(9,10)$. Therefore, it is essential that accurate activity uptake and related absorbed dose calculations be performed prior to therapy, to optimise the theragnostics' effectiveness by quantitative imaging. Consequently, inaccuracies in the image quantification process may result in incorrect absorbed dose calculations and can lead to a reduction in therapeutic treatment efficacy. Physical factors which can potentially affect the accuracy in the activity estimation from an image are, the isotope-collimator combination used, the accuracy and precision in the scatter and attenuation correction methods, and the compensation for the limited spatial resolution that results in partial volume effects (PVEs). 
High-energy (HE) collimators with thicker septa are needed when imaging ${ }^{131} \mathrm{I}$ due to the relatively high primary photon energy of $364.5 \mathrm{keV}$, used for imaging, and because of the otherwise high septal scatter and penetration from the emission of the $636.9 \mathrm{keV}$ and $722.9 \mathrm{keV}$ photons (11). However, ${ }^{123}$ I imaging can be performed with either a low energy high resolution (LEHR) or a medium energy (ME) collimator (12-14). The ME collimator reduces the septal penetration and scatter from the higher-energy photons of ${ }^{123}$ I, resulting in improved image contrast and more accurate activity quantification. However, when appropriate septal scatter and penetration corrections are applied during image reconstruction, imaging with a LEHR collimator may very well be a useful alternative to a ME collimator $(15,16)$.

Corrections for object scatter, non-homogenous attenuation and collimator detector response (CDR) as well as collimator-septal scatter and septal penetration, can be made by incorporating these effects in the camera/patient model, that is the essential part of any iterative reconstruction algorithm. The iterative maximum likelihood expectation maximisation (MLEM) algorithm and the closely related ordered subset expectation maximisation (OS-EM) algorithm are routinely used and typically available on most commercial SPECT/CT systems $(17,18)$. The basic steps in an iterative reconstruction algorithm are shown in Figure 1. The main assumption is that if starting with an initial estimate of the activity distribution (blank or uniform image), the imaging process and the radiation transport are modelled in a forward projection to obtain the expected SPECT projections. These expected projections are then compared to measured projections, and an error projection is calculated as either the difference or the ratios of the expected and measured projections. The error projection is then back projected to create an error image. Once sufficient angles are processed (all or a subset of angles depending on the reconstruction algorithm), the error image is then used to update the source distribution estimate. The process is repeated (iterates) until the ratios converge to unity (i.e. maximizing the likelihood). Because the expected and measured projections agree, it is assumed that the reconstructed images reflect the activity distribution in the patient and the process stops. However, the assumption is only valid if the same physical processes present in the real measurement are also modelled in the forward projection of the expected projection. 


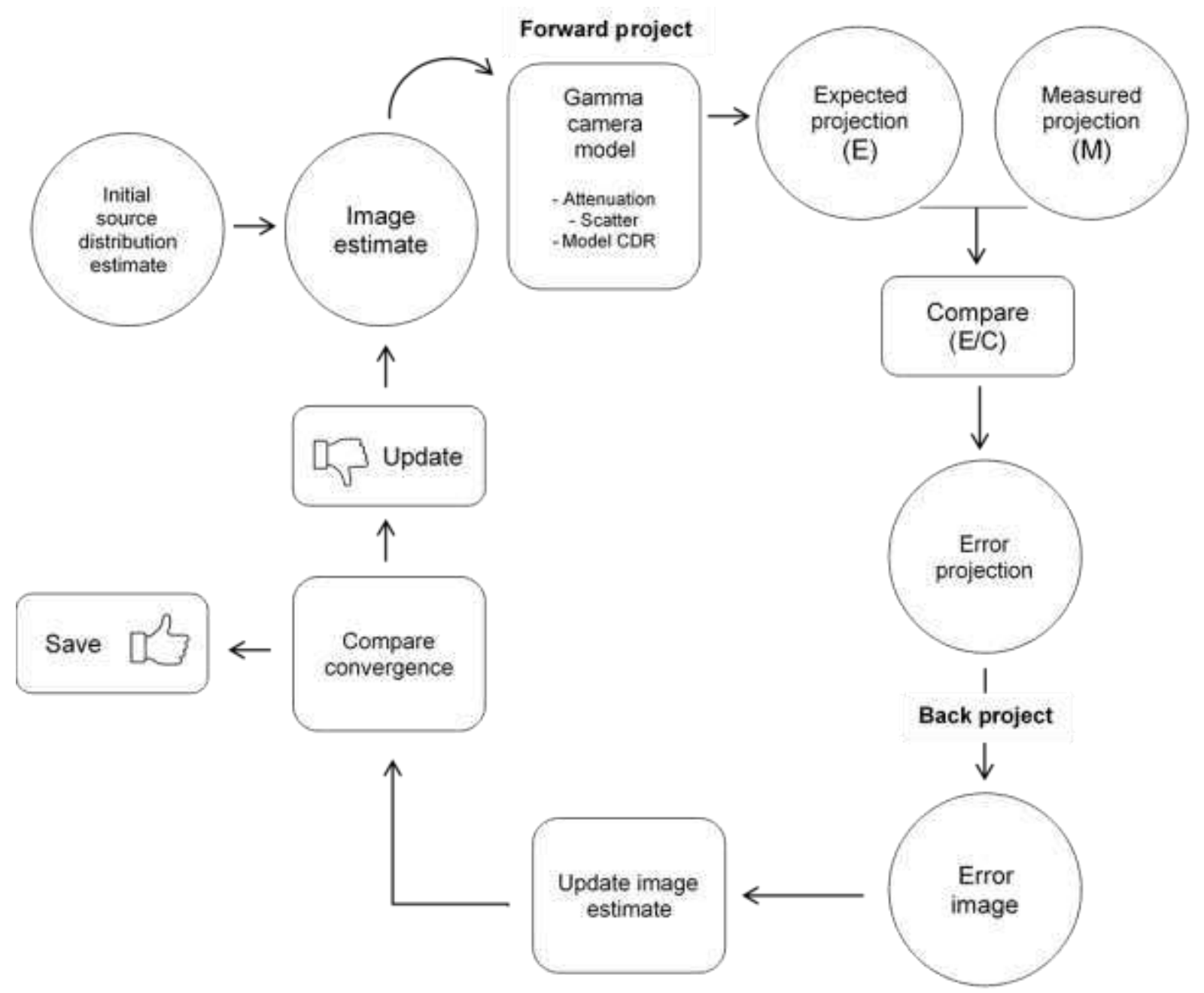

Figure 1: Schematic describing basic steps of iterative reconstruction method.

As the number of iterations increases, the reconstruction bias (difference between the estimated and measured data) is reduced; however, at the cost of image noise and reconstruction time. At a certain point, a further increase in the number of iterations will only increase image noise and reconstruction time (19). Thus, it is important to determine the optimal number of OS-EM updates (number of subsets multiplied by the number of iterations) prior to reconstructing SPECT images for activity quantification purposes.

Another prerequisite for absolute SPECT activity quantification (20) is the conversion of reconstructed image counts to activity or activity concentration, using a gamma camera conversion factor (CF). Different methods of establishing such conversion factors (CFs) have been suggested. The simplest method is a planar acquisition of a small point-like source in air, where self-attenuation within the source is kept minimal, and corrections for scatter have been applied (21). The gamma camera system sensitivity obtained from a petri-dish source in air, as defined by NEMA, has also been proposed (22). However, according to the definition of system 
sensitivity, counts in the entire image are used. This may be problematic for isotope-collimator combinations such as ${ }^{123} \mathrm{I}$ with the LEHR collimator and ${ }^{131}$ I with the HE collimator. For these isotopes-collimator combinations, a significant number of counts, originating from the higher energy photons which have penetrated the collimators' septa, are detected within the main photopeak energy window. It is generally believed that a CF obtained with a source geometry that mimics the scatter and attenuation conditions in a patient is better suited (11). SPECT acquisitions of geometries such as spheres in air or in water-filled cylindrical phantoms have been recommended, thereby reducing the effects of inadequate compensation for scatter and attenuation (20). Both planar-based and SPECT-based CF methods rely on accurate corrections for scatter and attenuation (23,24). Unlike for planar imaging, corrections for scatter, attenuation and CDR are now often implemented in clinical workstations and thus can be routinely performed on SPECT/CT image data sets, making the SPECT-based CF the method of choice. Considering that the same corrections are applied to both the clinical and the CF data set, any effects of imperfect correction methods would be minimised.

In SPECT imaging, the partial volume effect (PVE) is the result of the gamma camera's finite spatial resolution and the discretisation of the measured coordinates from the gamma camera to a digital image, and results in a reduction of image contrast between the regional uptake and surrounding areas. It is also a primary source-of-error when estimating activity in volumes-ofinterest (VOIs) with sizes that are in the order of the camera's resolution. Typically, VOIs smaller than three times the systems spatial resolution appear to contain less than actual activity concentrations (25). PVEs are only partially compensated for when SPECT images are reconstructed with CDR compensations. Therefore, a simple and effective method for the correction of residual PVEs is to use recovery coefficients (RCs) determined for objects of similar sizes as the VOIs. RCs are often expressed as the ratio of the estimated activity concentration in a VOI to the true activity concentration. RCs can be obtained from phantom measurements where the geometry of the sources and its activity concentrations are known or through Monte Carlo (MC) simulations, where RCs for more irregular and arbitrary shapes can be defined (26). A curve of RCs as a function of object size can be generated and a recovery coefficient (RC) value for a relevant VOI size can then be applied in the activity quantification process $(24,27-29)$. Often spherical sources are used to obtain RCs, but it should be noted that the RC also depends on the shape of the object (30). Since the variation of tumours can vary significantly, it is hard to obtain RC curves for every possible tumour shape. Instead, it is common to compensate for PVEs of non-spherical tumours by applying volume-specific RCs, 
which were determined from spherical phantoms, but, this method has been reported to have limitations (31).

The MC method is an important tool to evaluate the quantification accuracy and its effects on the accuracy of dosimetry, for a given SPECT/CT procedure. The MC modelling of gamma cameras for imaging of isotopes, including technetium-99m $\left({ }^{99 \mathrm{~m}} \mathrm{Tc}\right),{ }^{131} \mathrm{I}$ and lutetium-177 $\left({ }^{177} \mathrm{Lu}\right)$, have been reported extensively $(11,32,33)$. There are various MC simulation codes available for NM imaging (34-36). In this study we used the SIMIND MC code which models a standard clinical scintillation camera and has been successfully used to evaluate activity quantification accuracy (27,37-39). SIMIND allows for three methods to simulate the energy resolution, based on either (i) a simplified theoretical model assuming a $1 / \sqrt{E}$ dependence $(25,40)$, (ii) a fixed energy resolution used over the entire energy range, or (iii) a fitted model estimated from measured data (41). A fitted energy resolution model improves the energy resolution modelling of high-energy photon emitting isotopes (e.g., $\left.{ }^{131} \mathrm{I}\right)$ and isotopes with multiple photopeaks (e.g., ${ }^{123} \mathrm{I},{ }^{131} \mathrm{I}$ and ${ }^{177} \mathrm{Lu}$ ) and was therefore used in this study. SIMIND has been validated for simulating ${ }^{123} \mathrm{I}$ and ${ }^{131} \mathrm{I}$ images with the proposed fitted energy resolution model by Morphis et al. (41).

Although activity quantification accuracy with SIMIND has been reported in literature $(27,42,43)$, little work has been done using voxel-based digital phantoms for ${ }^{123} \mathrm{I}$ and ${ }^{131} \mathrm{I}$. Furthermore, to our knowledge, evaluating activity quantification accuracy in a clinically realistic phantom, using SIMIND with the fitted energy resolution model, has not yet been reported in literature.

The aim of the study was to evaluate SPECT activity quantification accuracy for ${ }^{123}$ I with both the LEHR and ME collimators, and ${ }^{131}$ I with the HE collimator, using the SIMIND MC code with a fitted energy resolution model. The objectives included (i) the optimisation of the number of OS-EM updates for the SPECT iterative reconstruction algorithm, (ii) determination of a $\mathrm{CF}$, (iii) determination of RC curves, and (iv) evaluation of the quantitative method in voxel-based digital simple and patient phantoms.

\section{Materials and Methods}

The Siemens Symbia T16 (Siemens Healthcare, Erlangen, Germany) dual-head SPECT/CT system with LEHR, ME and HE collimators, in use at Universitas Academic Hospital (Bloemfontein, South Africa), was modelled using version 6.2 of the SIMIND MC code (39). 
The setup of the virtual gamma camera (detector and collimators) and the simulation specifications were the same as that described and validated by Morphis et al. $(41,44)$.

Voxel-based digital phantoms were created from CT images of a water-filled cylindrical phantom (internal diameter: $20.3 \mathrm{~cm}$, external diameter: $21.6 \mathrm{~cm}$ and length: $31.7 \mathrm{~cm})(45)$, as well as from a randomly selected retrospective patient SPECT/CT data set from the Universitas Academic Hospital patient database. These voxel-based digital phantoms were segmented and created as described by Ramonaheng et al. $(27,46)$ and Morphis et al. $(41,44)$.

SPECT projections images were simulated for ${ }^{123} \mathrm{I}$ for both the LEHR and ME collimators (further referred to as ${ }^{123} \mathrm{I}$ LEHR and ${ }^{123} \mathrm{I} \mathrm{ME}$, respectively) and ${ }^{131} \mathrm{I}$ using the HE collimator $\left({ }^{131} \mathrm{I} \mathrm{HE}\right)$. As part of the simulation process, Poisson noise was added to the projection data. Energy windows of $15.0 \%$ were centred over the $159.0 \mathrm{keV}$ and $364.0 \mathrm{keV}$ photopeaks of ${ }^{123} \mathrm{I}$ and ${ }^{131} \mathrm{I}$, respectively. The images were simulated according to a standard clinical imaging protocol (step and shoot mode, non-circular orbit of rotation, 60 projections, 40 seconds per projection, matrix size: $128 \times 128$, pixel size: $4.8 \times 4.8 \mathrm{~mm}^{2}$ ). Simulations were performed with a high number of histories (> 1 billion) (43).

SPECT reconstruction was performed using software developed at Lund University, Sweden (47), which incorporates an OS-EM iterative reconstruction package, developed by Frey and Tsui (48). The iterative reconstruction algorithm performs a CT-based attenuation correction, model-based scatter correction by the Effective Scatter Source Estimation algorithm (ESSE), and a CDR correction, which accounts for collimator septal penetration and scatter by using pre-calculated MC simulated kernels (49). The SPECT images were reconstructed in a $128 \times 128 \times 128$ matrix with a voxel size of $4.8 \times 4.8 \times 4.8 \mathrm{~mm}^{3}$. Reconstructed image count statistics (total, mean and standard deviations) were obtained using the public domain software, Amide (50).

\subsection{Optimisation of OS-EM updates}

When determining the optimal number of OS-EM updates, it is essential to consider the tradeoff between bias, image noise and reconstruction time. To determine the optimal number of OS-EM updates and evaluate activity recovery as a function of the number of OS-EM updates, four spheres, with volumes of 1.7, 14.1, 47.7 and $113.1 \mathrm{~mL}$ (diameters: 1.5, 3.0, 4.5 and $6.0 \mathrm{~cm}$ ), were digitally added to a segmented image of the water-filled cylindrical phantom. 
Different sized spheres were chosen to ensure coverage of at least one to four times the planar spatial resolution of ${ }^{131} \mathrm{I}$ when imaged with the $\mathrm{HE}$ collimator (spatial resolution of $1.3 \mathrm{~cm}$ ).

SPECT projections were simulated, with simulation parameters as explained above, for ${ }^{123} \mathrm{I}$ and ${ }^{131} \mathrm{I}$, using sphere activity concentration values of $0.7 \mathrm{MBq} / \mathrm{mL}$ and $1.1 \mathrm{MBq} / \mathrm{mL}$, respectively. The concentration values were based on clinical ${ }^{123} \mathrm{I}$ and ${ }^{131} \mathrm{I}$ mIBG tumour data at approximately 24 hours post-administration. The SPECT projections were reconstructed using LundADose, as described above. A series of 17 reconstructions were performed for each isotope-collimator combination, with six subsets, as proposed in literature $(11,19)$, and OS-EM updates varying from 18 to 168. Count statistics (expressed as counts per second per mL) were obtained for each reconstructed sphere, with spherical VOIs equal to the sphere's physical size. Each VOI value was normalised to the overall maximum VOI value (33).

Brambilla et al. (51) and Leong et al. (52) evaluated reconstruction noise by assessing the change in the reconstruction noise level within a uniform area of a single slice. However, Ramonaheng et al. (27) suggested assessing the change in reconstruction noise level within a target VOI, obtained using the object's physical size. This provides a constant and reliable delineation method, which is the same as that used to assess count recovery. As proposed by Ramonaheng et al. (27), the reconstruction noise levels in each sphere were calculated using Equation 1,

$$
\mathrm{SD}_{\text {relative }}=\frac{S D}{\text { mean }} \times 100
$$

where $\mathrm{SD}_{\text {relative }}$ is the relative standard deviation, $S D$ and mean are the standard deviation and mean counts per voxel of the reconstructed counts from the VOI of the sphere. It is important to note that $\mathrm{SD}_{\text {relative }}$ is only an estimation of reconstruction noise (53). Furthermore, in scenarios where CDR correction is included in the iterative reconstruction, the effect of the Gibbs artifact increases with an increase in the number of iterations, thus the $\mathrm{SD}_{\text {relative }}$ will include this variation.

The normalised recovered counts and $\mathrm{SD}_{\text {relative }}$ values per sphere size were plot as a function of the number of OS-EM updates. The optimal number of OS-EM updates was determined from the recovered counts based on the graphs for each isotope-collimator combination and used in the subsequent reconstructions. 
The optimal number of OS-EM updates was confirmed in a clinically realistic voxel-based digital patient phantom, where the activity recovery in a $14.1 \mathrm{~mL}$ (diameter: $3.0 \mathrm{~cm}$ ) sphere was evaluated at several of the OS-EM updates.

\subsection{Conversion factors}

For each isotope-collimator combination, four different source geometries were used to determine the gamma camera SPECT CFs. The source geometries included an activity-filled cylindrical phantom $\left(\mathrm{CF}_{\text {cylinder }}\right)$, a $113.1 \mathrm{~mL}$ (diameter: $6.0 \mathrm{~cm}$ ) activity-filled sphere in the water-filled (cold) cylindrical phantom $\left(\mathrm{CF}_{\text {water }}\right)$, a $113.1 \mathrm{~mL}$ activity-filled sphere in air $\left(\mathrm{CF}_{\text {air }}\right)$ and a point-like source (volume $<1 \mathrm{~mL})$ in air $\left(\mathrm{CF}_{\text {point }}\right)$. Concentration values for the four different $\mathrm{CF}$ source geometries were the same as the concentration value used to determine the optimal number of OS-EM updates.

SPECT projection images were simulated with the phantoms placed at the centre of the gamma camera's field of view and reconstructed using the optimised reconstruction parameters. Count statistics were obtained for each reconstructed CF source geometry. Cylindrical or spherical VOIs were defined based on the $\mathrm{CF}$ source geometry (cylindrical for $\mathrm{CF}_{\text {cylinder }}$ and spherical for the remaining geometries). Multiple VOI sizes with diameters of (i) less than the physical size, (ii) the physical size plus a $3.0 \mathrm{~cm}$ margin or (iii) the physical detector size, were defined.

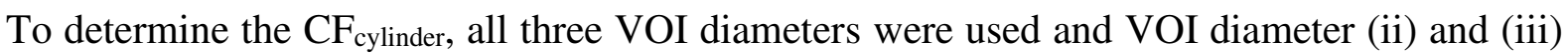
were chosen for the remaining CFs. Due to the presence of Gibbs artefacts in the $\mathrm{CF}_{\text {water }}$ and $\mathrm{CF}_{\text {air }}$ images, and to the limited size of the point source for $\mathrm{CF}_{\text {point }}$, VOIs smaller than the sources physical size were not considered. The CFs were calculated using Equation 2,

$$
C F=\frac{C n t}{A \times t}\left(\frac{c p s}{M B q}\right)
$$

where $C n t$ is the total image counts obtained in each VOI, $A$ is the simulated activity in the source, and $t$ the simulated acquisition time. From this, an appropriate CF source geometry was determined for all isotope-collimator combinations.

Energy spectra were also obtained from the $\mathrm{CF}_{\text {point }}$ SPECT simulations, for each isotopecollimator combination, to determine any possible contributions of septal scatter and penetration to the CFs. 


\subsection{Recovery coefficient curves}

RCs were determined for the dual-head Siemens Symbia T16 SPECT/CT gamma camera. Eight spheres, with volumes ranging from 8.2 to $523.6 \mathrm{~mL}$ (diameters: 2.5 to $10.0 \mathrm{~cm}$ ), were digitally added to the segmented images of the water-filled cylindrical phantom. SPECT projections were simulated, with sphere activity concentration values and simulation parameters as explained above. Simulations were performed without background activity in the cylindrical phantom, to avoid any biased results in the quantification accuracy. The SPECT projections were reconstructed using the optimised reconstruction parameters. Count statistics with VOIs defined by the physical sphere size were obtained for each sphere using the predetermined CF geometry, and reported in units of $\mathrm{MBq} / \mathrm{mL}$. The recovered and true activity concentration $\left([A]_{\text {recovered }}\right.$ and $\left.[A]_{\text {true }}\right)$ values were used to calculate the RCs, according to Equation 3,

$$
R C=\frac{[A]_{\text {recovered }}}{[A]_{\text {true }}}
$$

The RCs for each isotope-collimator combination were plotted as a function of sphere diameter and fitted with a mono-exponential function, as shown in Equation 4, proposed by Willowson et al. (24),

$$
R C=a-b e^{-c x}
$$

where $x$ represents the sphere diameter $(\mathrm{cm})$, and $a, b$ and $c$ are the curve fitting parameters.

\subsection{Quantification}

\subsubsection{Spheres in the cylindrical phantom}

To evaluate the quantitative method, the error of the estimated activity in spheres (volumes ranging from 8.2 to $523.6 \mathrm{~mL}$ ) in an activity-filled cylindrical phantom was determined. Activity concentration values were based on clinical ${ }^{123} \mathrm{I}$ and ${ }^{131} \mathrm{I}$ mIBG pharmacokinetic data observed at 24-hours post-injection (sphere to background ratio of 100:1). The recovered activity concentration was calculated using the predetermined CF and corrected for PVE using RCs obtained from the RC curve, for each isotope-collimator combination. The quantification error was defined as the percentage difference between the recovered activity concentration $\left([A]_{\text {recovered }}\right)$ and the true activity concentration $\left([A]_{\text {true }}\right)$ defined in SIMIND, shown in Equation 5,

$$
\text { Quantification Error }(\%)=\frac{[A]_{\text {recovered }}-[A]_{\text {true }}}{[A]_{\text {true }}} \times 100
$$




\subsubsection{Voxel-based digital patient phantoms}

Two different sized spheres were added to a voxel-based digital patient phantom, to determine the accuracy in a clinically realistic phantom. As shown in Figure 2, two scenarios were considered; scenario 1 included a sphere with a diameter of $5.0 \mathrm{~cm}$ positioned above the liver between the lungs, and a second sphere with a diameter of $3.0 \mathrm{~cm}$ positioned below the liver. For scenario 2, the positions of the two spheres were switched. Activity concentration values were based on clinical ${ }^{123} \mathrm{I}$ and ${ }^{131} \mathrm{I}$ mIBG pharmacokinetic data observed at 24-hours postinjection (sphere to background ratio of 100:1, sphere to liver ratio of 100:7.5 and sphere to lung ratio of 100:2.8).

${ }^{123}$ I and ${ }^{131}$ I SPECT projections were simulated and reconstructed as previously described. Count statistics were obtained with VOIs defined according to the physical size of the spheres and the liver. Sphere and liver activity concentrations were calculated using the predetermined $\mathrm{CF}$ geometry and corrected for PVE using the appropriate $\mathrm{RC}$ obtained from the $\mathrm{RC}$ curve, and the error in the recovered activity concentration was determined using Equation 5.

(a)

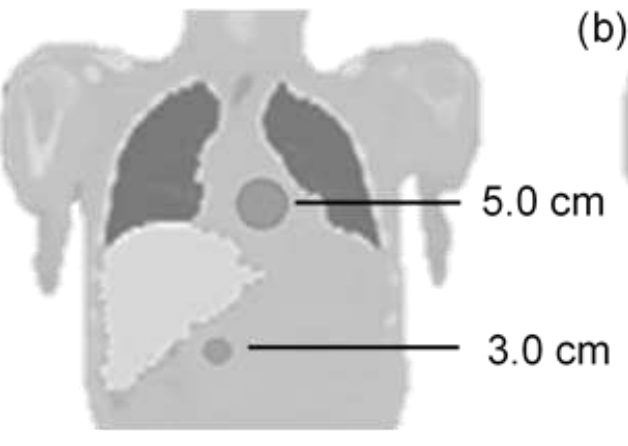

(b)

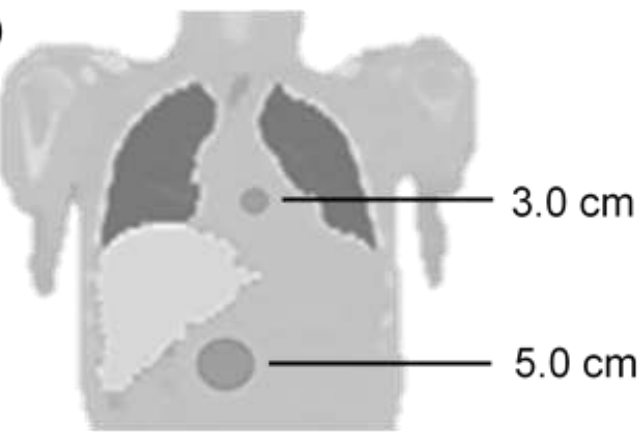

Figure 2: Segmented coronal CT slices of the voxel-based digital patient phantom with spheres' positions for (a) scenario 1 and (b) scenario 2.

\section{Results}

\subsection{Optimisation of OS-EM updates}

The relationship between the number of OS-EM updates and (i) the recovered counts and (ii) $\mathrm{SD}_{\text {relative }}$, for each sphere size is illustrated in Figure 3 for all isotope-collimator combinations.

Figure 3 (a), (b) and (c) show that count convergence (fast increase followed by a slower approach towards an asymptote) is reached relatively quickly for the three largest spheres, compared to the smallest sphere. Eighteen OS-EM updates resulted in more than $90 \%$ count recovery for the two larger spheres $(4.5 \mathrm{~cm}$ and $6.0 \mathrm{~cm})$, for all isotope-collimator 
combinations. However, more OS-EM updates were required to reach count convergence for the two smallest spheres $(1.5 \mathrm{~cm}$ and $3.0 \mathrm{~cm})$. This can be attributed to the limited spatial resolution and the PVE of ${ }^{123} \mathrm{I}$ LEHR, ${ }^{123} \mathrm{I} \mathrm{ME}$ and ${ }^{131} \mathrm{I} \mathrm{HE}$ (16).

The graphs in Figure 3 show that for all isotope-collimator combinations reaching 60 OS-EM updates, a further increase in OS-EM updates would not improve count recovery by more than $3.0 \%$ for the three larger spheres and $16.0 \%$ for the smallest sphere. The change in $\mathrm{SD}_{\text {relative }}$ from 18 OS-EM updates to 60 OS-EM updates was less than $8.0 \%$ for the three larger spheres. The smallest sphere resulted in a $\mathrm{SD}_{\text {relative }}$ increase of $15.0 \%$ (improved count recovery of $16.0 \%$ ), $16.0 \%$ (improved count recovery of 23.0\%) and $25.0 \%$ (improved count recovery of $26.0 \%$ ) for ${ }^{123} \mathrm{I}$ LEHR, ${ }^{123} \mathrm{I} \mathrm{ME}$ and ${ }^{131} \mathrm{I} \mathrm{HE}$, respectively. With the goal of accurate activity quantification, an improvement in count recovery was favoured. Based on these considerations, 60 OS-EM updates were considered optimal for all isotope-collimator combinations. This was confirmed by the recovered activity in the $3.0 \mathrm{~cm}$ sphere (mimicking a tumour) in the voxelbased digital patient phantom. A further increase in the number of OS-EM updates did not improve count recovery by more than $4.7 \%$. 
(a) ${ }^{123}$ LEHR

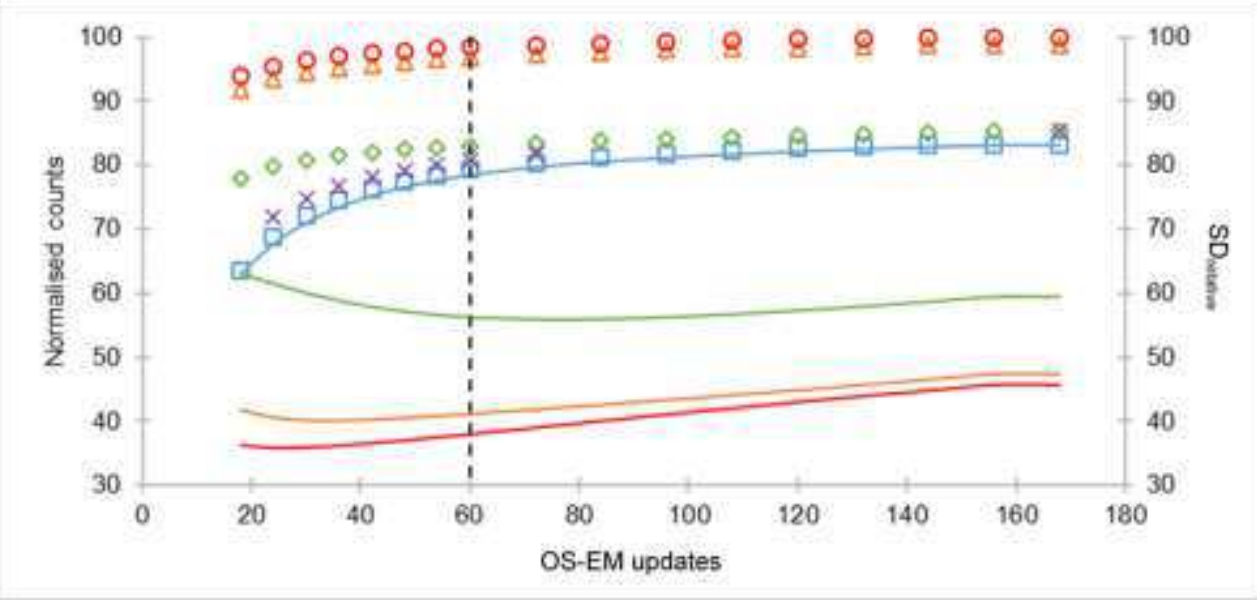

(b) ${ }^{123} \mathrm{ME}$

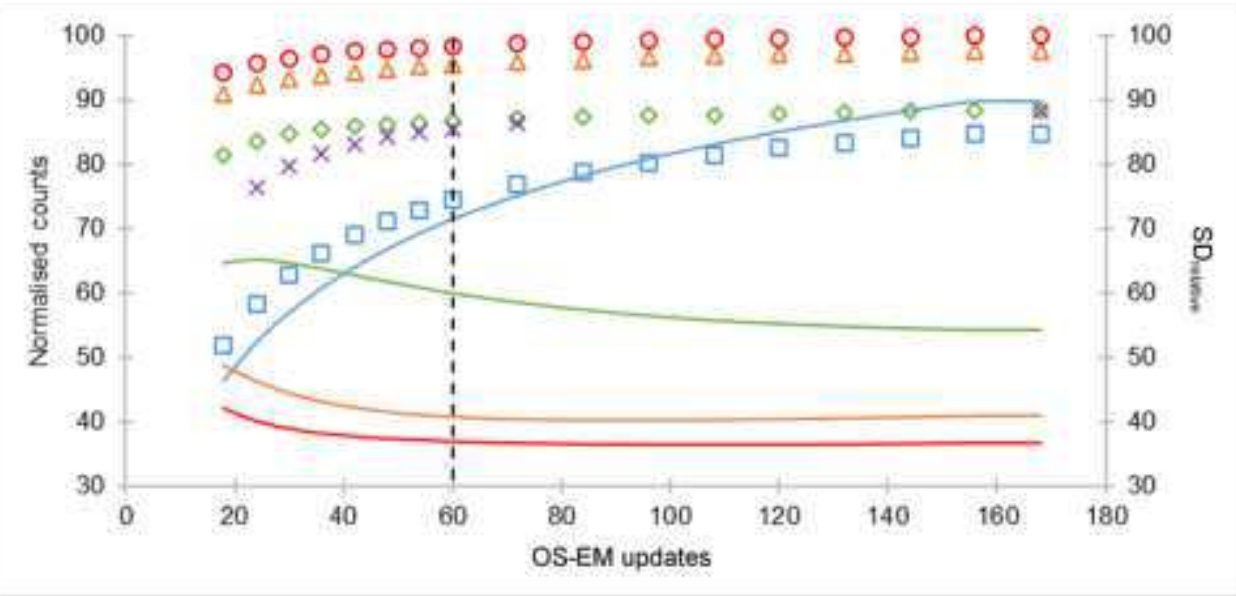

(c) ${ }^{131} \mathrm{IHE}$

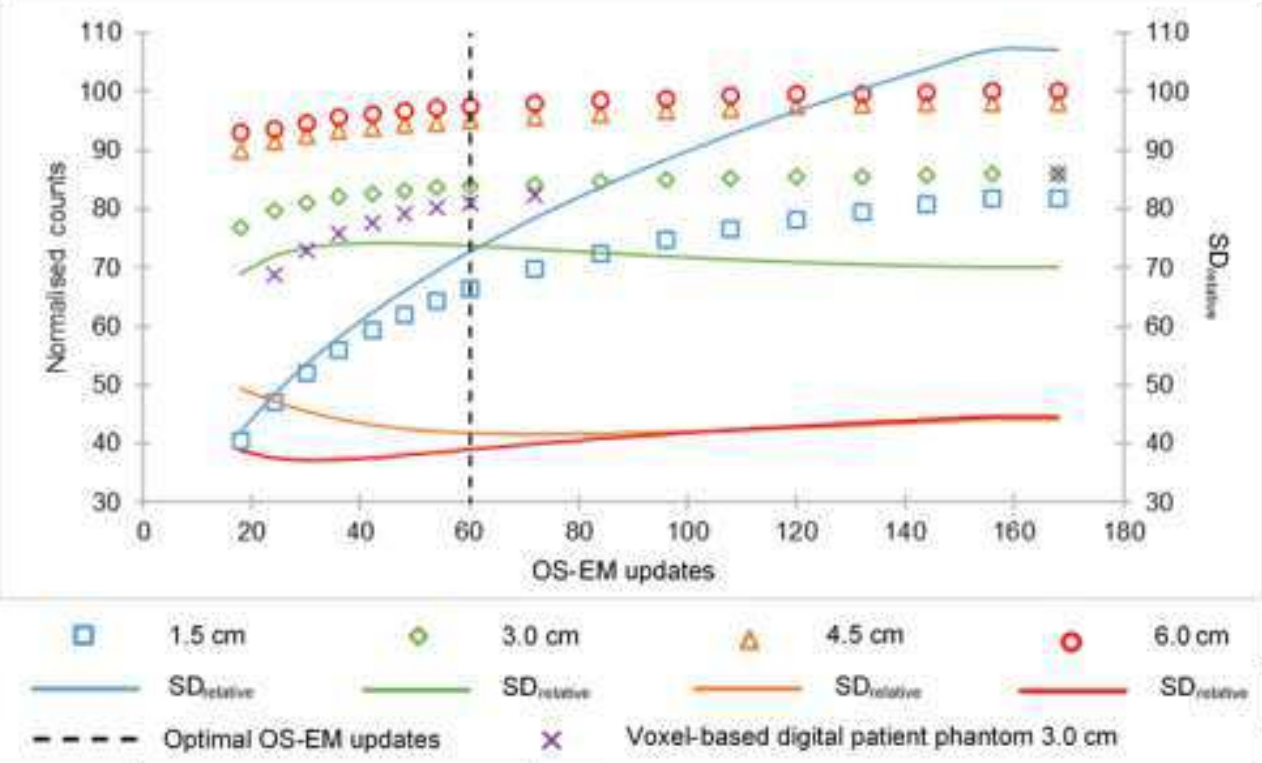

Figure 3: Normalised counts and $\mathrm{SD}_{\text {relative }}$ as a function of OS-EM updates for spheres with diameters of $1.5 \mathrm{~cm}, 3.0 \mathrm{~cm}, 4.5 \mathrm{~cm}$, and $6.0 \mathrm{~cm}$, for (a) ${ }^{123} \mathrm{I} \mathrm{LEHR,} \mathrm{(b)}{ }^{123} \mathrm{I} \mathrm{ME}$ and (c) ${ }^{131} \mathrm{I} \mathrm{HE}$. 


\subsection{Conversion factors}

Table 1 shows the CFs for the different source geometries and VOI diameters. The relative SDs for the average of the four CFs, when selecting a VOI diameter equal to the physical source diameter plus $3.0 \mathrm{~cm}$, were $2.9 \%, 1.1 \%$ and $1.5 \%$ for ${ }^{123} \mathrm{I} \mathrm{LEHR},{ }^{123} \mathrm{I} \mathrm{ME}$ and ${ }^{131} \mathrm{I} \mathrm{HE}$, respectively. Similar results were obtained when selecting the VOI equal to the detector's physical size (relative SDs of $1.9 \%, 0.8 \%$ and $4.7 \%$ for ${ }^{123} \mathrm{I}$ LEHR, ${ }^{123} \mathrm{I}$ ME and ${ }^{131} \mathrm{I} \mathrm{HE}$, respectively). For ${ }^{123}$ I LEHR, the selection of the different VOI margins resulted in a $5.0 \%$ difference in CF between VOI selection (i) and (ii), $13.0 \%$ difference in CF between VOI selection (i) and (iii) and 19.0\% difference in CF between VOI selection (ii) and (iii). The corresponding differences obtained for ${ }^{123} \mathrm{I} \mathrm{ME}$ were all less than 2.2\%. ${ }^{131} \mathrm{I}$ HE resulted in differences of $1.1 \%, 7.2 \%$ and $8.4 \%$, respectively. These results show that the effect of VOI selection is more pronounced for ${ }^{123} \mathrm{I}$ LEHR and ${ }^{131} \mathrm{I}$ HE. This can be attributed to unwanted septal scatter and penetration, which was not fully corrected for using the OS-EM reconstruction and the appropriate correction methodologies.

\section{Table 1}

CFs for ${ }^{123} \mathrm{I}$ with the LEHR and ME collimator and ${ }^{131} \mathrm{I}$ with the HE collimator, in cps/MBq, for the four CF source geometries. VOI diameters were defined as (i) less than the physical source diameter, (ii) equal to the physical source diameter plus a $3 \mathrm{~cm}$ margin, and (iii) equal to the physical size of the detector.

\begin{tabular}{|c|c|c|c|c|}
\hline $\begin{array}{l}\text { VOI } \\
\text { diameter }\end{array}$ & & $\begin{array}{l}{ }^{123} \text { I LEHR } \\
\text { (cps/MBq) }\end{array}$ & $\begin{array}{l}{ }^{123} \mathrm{I} \mathrm{ME} \\
(\mathrm{cps} / \mathrm{MBq})\end{array}$ & $\begin{array}{l}{ }^{131} \mathrm{I} \mathrm{HE} \\
(\mathrm{cps} / \mathrm{MBq})\end{array}$ \\
\hline i & $\mathrm{CF}_{\text {cylinder }}$ & 89.3 & 119.0 & 27.7 \\
\hline \multirow{6}{*}{ ii } & $\mathrm{CF}_{\text {cylinder }}$ & 88.4 & 115.6 & 26.8 \\
\hline & $\mathrm{CF}_{\text {water }}$ & 84.4 & 118.2 & 27.6 \\
\hline & $\mathrm{CF}_{\text {air }}$ & 82.4 & 116.4 & 27.2 \\
\hline & $\mathrm{CF}_{\text {point }}$ & 84.1 & 115.5 & 27.8 \\
\hline & Average \pm SD & $84.8 \pm 2.5$ & $116.4 \pm 1.3$ & $27.4 \pm 0.4$ \\
\hline & Relative SD (\%) & 2.9 & 1.1 & 1.5 \\
\hline \multirow{4}{*}{ iii } & $\mathrm{CF}_{\text {cylinder }}$ & 100.4 & 118.9 & 28.6 \\
\hline & $\mathrm{CF}_{\text {water }}$ & 102.2 & 120.2 & 29.1 \\
\hline & $\mathrm{CF}_{\mathrm{air}}$ & 98.4 & 117.7 & 29.4 \\
\hline & $\mathrm{CF}_{\text {point }}$ & 102.4 & 118.7 & 31.7 \\
\hline
\end{tabular}




\begin{tabular}{llll}
\hline Average \pm SD & $100.9 \pm 1.9$ & $118.9 \pm 1.0$ & $29.7 \pm 1.4$ \\
Relative SD (\%) & 1.9 & 0.8 & 4.7 \\
\hline
\end{tabular}

To better visualise the effect of septal scatter and penetration, simulated energy spectra of $\mathrm{CF}_{\text {point }}$ projection images are shown in Figure 4. Higher levels of septal penetration (indicated by the arrows) can be seen in Figure 4 (a) compared to Figure 4 (b) and (c). This further reiterates the importance of accurate septal scatter and penetration corrections, which could be the cause of the larger difference between CFs with VOI diameters (i) and (ii) to that of (iii) for ${ }^{123}$ I LEHR, as shown in Table 1.
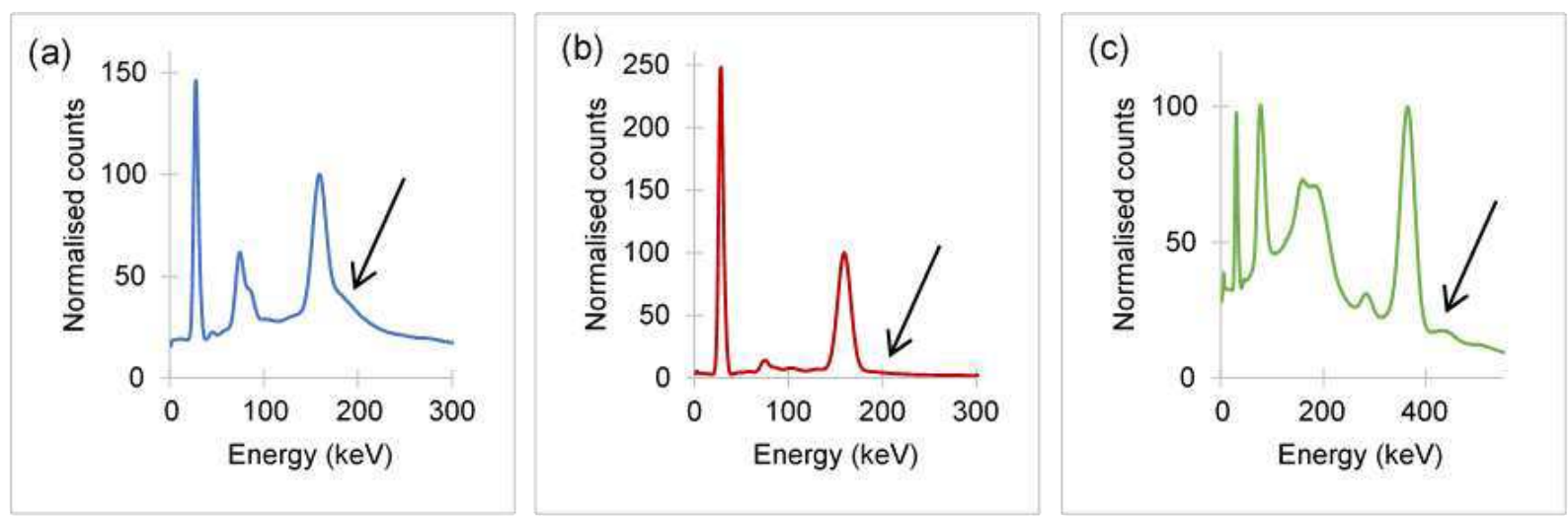

Figure 4: Simulated energy spectra of $\mathrm{CF}_{\text {point }}$ projection images for (a) ${ }^{123} \mathrm{I}$ LEHR, (b) ${ }^{123} \mathrm{I} \mathrm{ME}$, and (c) ${ }^{131} \mathrm{I} \mathrm{HE}$.

Based on these findings, the $\mathrm{CF}_{\text {point }}$ with a VOI diameter equal to the physical size plus a $3.0 \mathrm{~cm}$ margin, representing the simplest geometry, was considered appropriate to use as a CF for all isotope-collimator combinations.

\subsection{Recovery coefficient curves}

Figure 5 shows the calculated RCs as a function of sphere diameter for all isotope-collimator combinations. The mono-exponential functions fitted to the data, allowing for interpolation between sphere sizes, had $\mathrm{R}^{2}$ values of 0.99 for all isotope-collimator combinations. From Figure 5 it is evident that for ${ }^{123}$ I LEHR and ${ }^{123} \mathrm{I} \mathrm{ME}$, more than $90.0 \%$ (RC > 0.9) of the activity concentration is recovered for spherical sources with diameters equal to and larger than $5.0 \mathrm{~cm}$. Comparatively, more than $80.0 \%$ ( $\mathrm{RC}>0.8$ ) of the activity concentration was recovered for ${ }^{131} \mathrm{I} \mathrm{HE}$ at sphere diameters of $5.0 \mathrm{~cm}$ and more, and $90.0 \%(\mathrm{RC}>0.9)$ at sphere diameters equal to and larger than $7.0 \mathrm{~cm}$. This emphasises the impact of PVEs for isotope-collimator combinations with poorer spatial resolution, such as ${ }^{131} \mathrm{I} \mathrm{HE}$. 


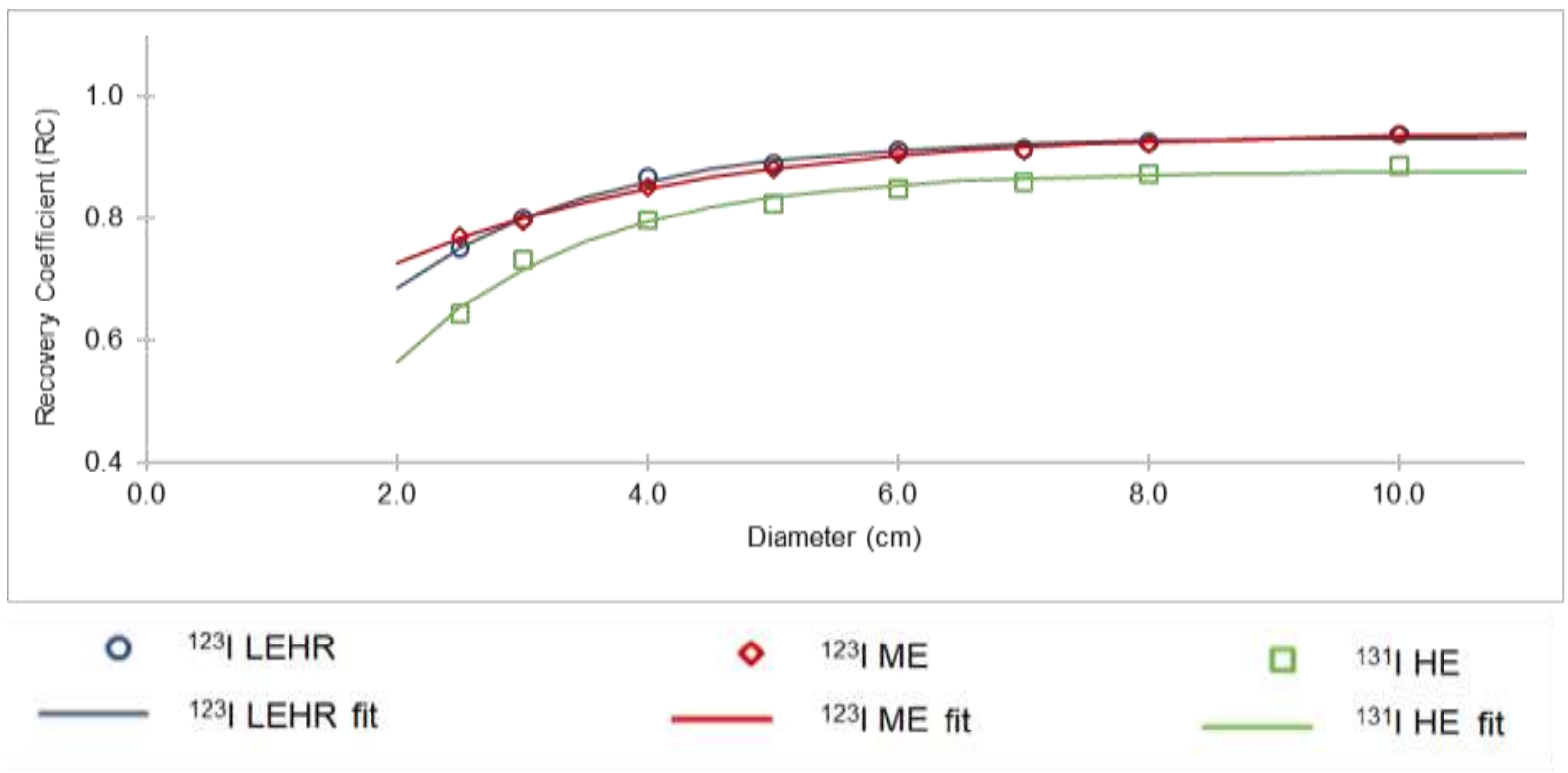

Figure 5: RC as a function of sphere diameter for ${ }^{123} \mathrm{I}$ LEHR, ${ }^{123} \mathrm{I} \mathrm{ME}$ and ${ }^{131} \mathrm{I} \mathrm{HE}$, with the solid lines showing the fitted functions.

\subsection{Quantification}

\subsubsection{Spheres in the cylindrical phantom}

The quantification error, as defined by Equation 5, for spheres with diameters ranging from 2.5 to $10.0 \mathrm{~cm}$, in a warm cylindrical phantom, obtained with the $\mathrm{CF}$ and recovery curves, as determined in Sections 3.2 and 3.3, respectively, for all isotope-collimator combinations, is shown in Figure 6. Overall, percentage quantification errors were less than $3.2 \%$ for all isotopecollimator combinations.

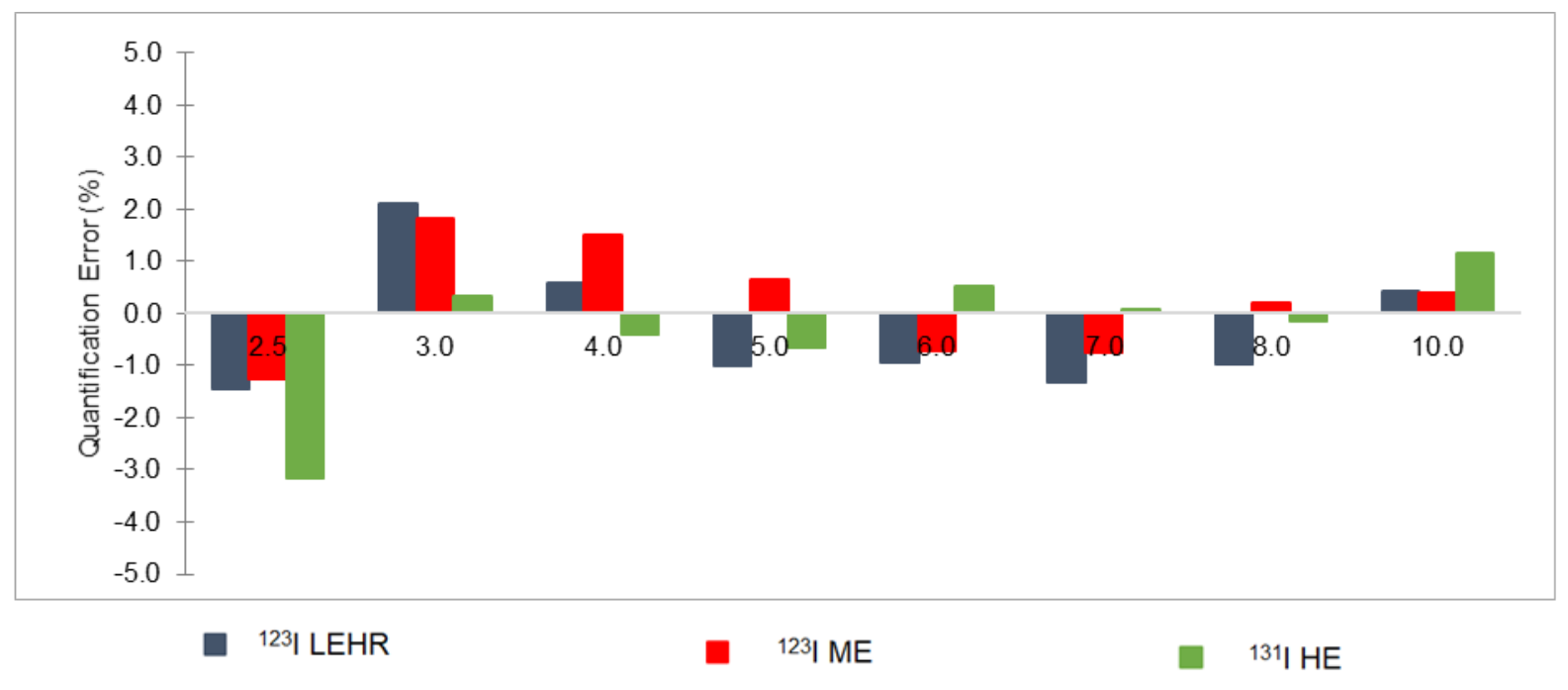

Figure 6: Quantification error (\%) of spheres of differing diameter in a warm cylindrical phantom, for ${ }^{123} \mathrm{I}$ LEHR, ${ }^{123} \mathrm{I} \mathrm{ME}$ and ${ }^{131} \mathrm{I} \mathrm{HE}$. 


\subsubsection{Voxel-based digital patient phantoms}

Figure 7 shows projection images and reconstructed transaxial slices of the voxel-based digital patient phantom for all isotope-collimator combinations. Additionally, profiles through the largest sphere (diameter: $5.0 \mathrm{~cm}$ ) are shown alongside their respective images.

Differences in background counts due to collimator septal scatter and penetration can be seen between ${ }^{123}$ I LEHR and ${ }^{123}$ I ME in Figure 7 (a) and Figure 7 (c), respectively. The presence of septal scatter and penetration is further illustrated with the profile through the $5.0 \mathrm{~cm}$ sphere, as shown by the arrows in Figure 7 (a) compared to Figure 7 (c). However, comparing the reconstructed slices and the profile through the $5.0 \mathrm{~cm}$ sphere, as shown in Figure 7 (b) and Figure 7 (d), it can be seen that septal scatter and penetration is sufficiently compensated for the case of ${ }^{123}$ I LEHR (ratio of peak to trough counts increased). Overall, the ${ }^{123}$ I LEHR images appear slightly noisier compared to ${ }^{123} \mathrm{I}$ ME. This can be attributed to poorer counts statistics due to the reduced sensitivity of ${ }^{123}$ I LEHR compared to ${ }^{123} \mathrm{I} \mathrm{ME}$, considering equal amounts of activity were simulated in each. The effects of septal scatter and penetration are notably lower for ${ }^{131} \mathrm{I} \mathrm{HE}$, as shown in Figure 7 (e) and Figure 7 (f). Visually, the ${ }^{123} \mathrm{I}$ ME images are comparable to the ${ }^{131}$ I HE images. Similar differences have been reported in literature by Nakajima et al. (54). 

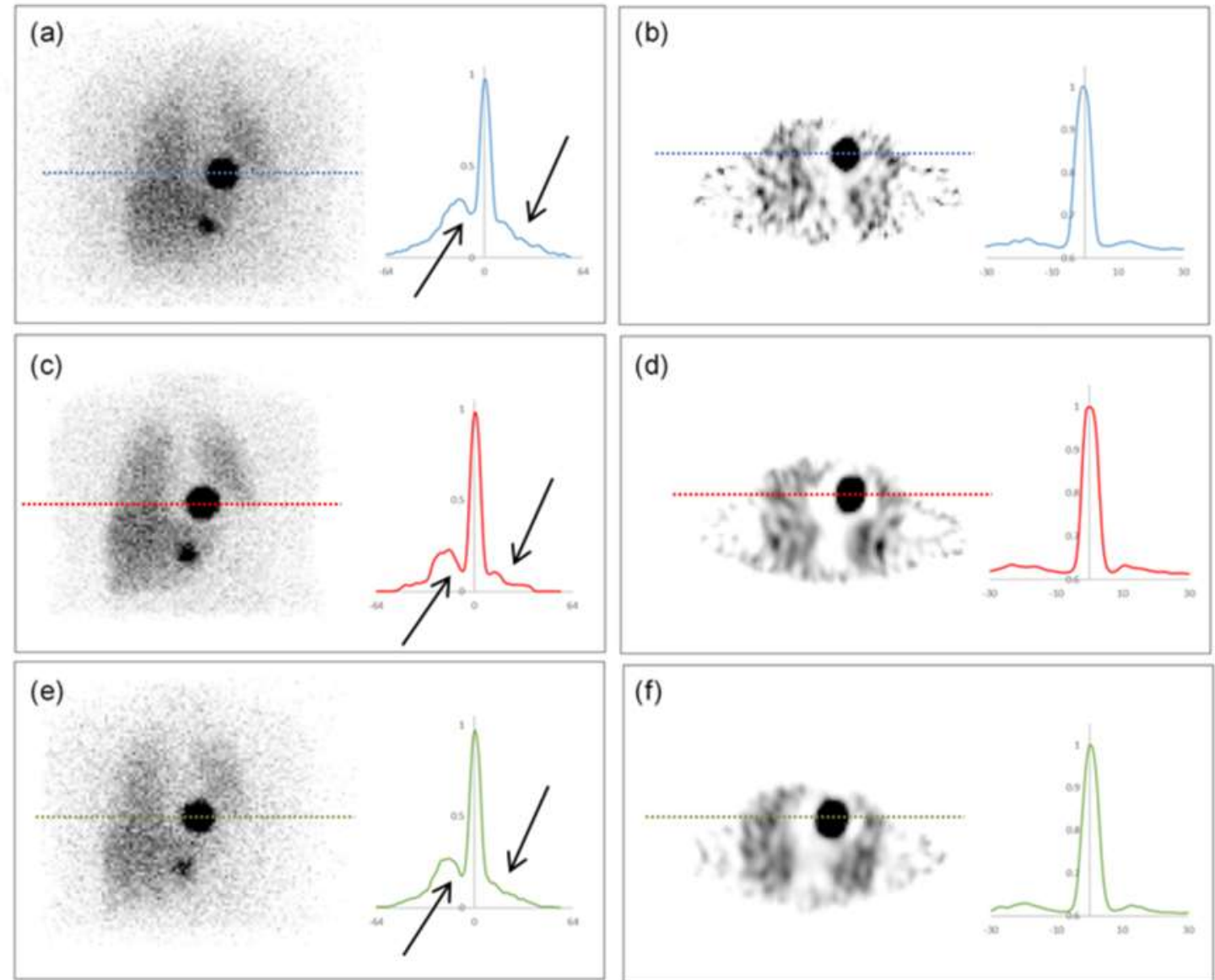

Figure 7: Uncorrected projection images (a, c, e), and reconstructed corrected transaxial slices (b, d, f) through the voxel-based digital patient phantom for (a, b) ${ }^{123}$ I LEHR, (c, d) ${ }^{123}$ I ME, and $(e, f){ }^{131}$ I HE. Profiles through the largest sphere are displayed alongside their respective images. Arrows indicate septal scatter and penetration at peak and trough and at tail region.

The activity quantification errors in the two spheres are shown in Figure 8 for all isotopecollimator combinations. The $3.0 \mathrm{~cm}$ and $5.0 \mathrm{~cm}$ spheres show an absolute quantification error of less than $5.4 \%$ for all isotope-collimator combinations for both scenarios 1 and 2 . For the liver, absolute quantification errors of less than $5.2 \%$ are noted across all isotope-collimator combinations. 


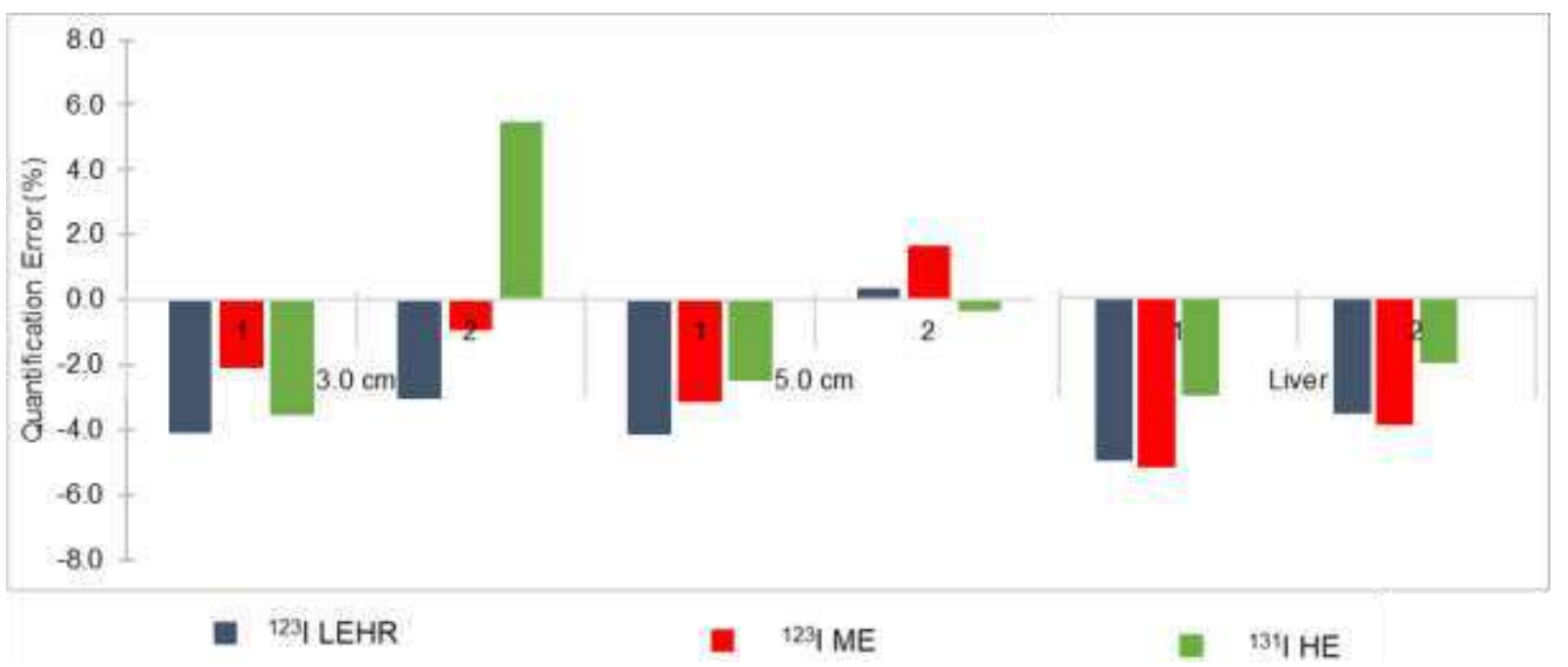

Figure 8: Activity quantification errors (\%) for spheres in the voxel-based digital patient phantom (scenario 1 and 2), for ${ }^{123}$ I LEHR, ${ }^{123} \mathrm{I} \mathrm{ME}$, and ${ }^{131} \mathrm{I}$ HE.

\section{Discussion}

The purpose of our work was to evaluate the accuracy of activity quantification for SPECT imaging when using ${ }^{123} I$ with both the LEHR and ME collimators, and for ${ }^{131} I$ with the HE collimator.

\section{Optimisation of OS-EM updates}

From the results, it is evident that optimisation of the number of OS-EM updates is important for accurate SPECT activity quantification. Literature states that smaller objects require a larger number of OS-EM updates to improve reconstruction accuracy (19), which corroborates our results, as shown in Figure 3.

It is well known that increasing the number of OS-EM, increases image noise. Literature supports this statement for several isotopes, including ${ }^{99 \mathrm{~m}} \mathrm{Tc}(52),{ }^{131} \mathrm{I}(43,55,56)$ and ${ }^{177} \mathrm{Lu}$ $(27,57,58)$. The results in Figure 3 corroborate that stated in literature, showing that the image of the smallest sphere has the largest $\mathrm{SD}_{\text {relative }}$ value, and that the value decreases as the sphere size increases.

Figure 3 also shows that the optimal number of OS-EM updates is object size-dependent. Based on these findings, the optimal number of OS-EM updates was found to be 60 for all isotopecollimator combinations. Our study's findings were similar to results reported in the literature $(11,19,59)$. The choice of optimal number of OS-EM updates was confirmed by similar convergence obtained with the voxel-based digital patient phantom (Figure 3). 


\section{Conversion factors}

Literature reports the use of several CFs, which include the activity-filled cylindrical phantom, point-like or spherical source in air $(20,21)$. The simplest way of determining a CF is from a point-like source in-air. However, this method relies on SPECT data which has been reconstructed with high accuracy. To account for any uncertainties in the reconstruction process, a more reliable source geometry is one which better approximates the patients' scatter and attenuation properties (43). However, Dewaraja et al. (43), also stated that when comparing ${ }^{131}$ I HE quantification results obtained with a spherical (volume: $200.0 \mathrm{ml}$, diameter: $7.3 \mathrm{~cm}$ ) and a point source $\mathrm{CF}$, there was no evidence that one geometry outperformed the other.

As with D'Arienzo et al. (20), the relative percentage SD values displayed in Table 1 show little difference between the four $\mathrm{CF}$ source geometries $\left(\mathrm{CF}_{\text {cylinder }}, \mathrm{CF}_{\text {water }}, \mathrm{CF}_{\text {air }}\right.$ and $\left.\mathrm{CF}_{\text {point }}\right)$. However, this small error may not always be representative, as the error is highly dependent on the compensation methods implemented in the clinical reconstruction algorithm. The reconstruction algorithm used in this study includes compensation for object scatter and attenuation as well as CDR which, in turn, compensates partly for collimator septal scatter and penetration. This is not always true for software implemented on clinical workstations.

Our results showed the importance of the choice of VOI size when obtaining a CF. This is especially important when imaging isotope-collimator combinations resulting in high levels of septal scatter and penetration, as seen in Figure 4 (a) and (c) for ${ }^{123}$ I LEHR and ${ }^{131} \mathrm{I}$ HE, respectively. Despite the reconstruction process accounting for septal scatter and penetration, counts from septal scatter and penetration counts are still present, as evident in the results shown in Table 1. The difference in CF when using VOI diameters (ii) and (iii) is $18.9 \%$ and $8.6 \%$ for ${ }^{123}$ I LEHR and ${ }^{131}$ I HE, respectively. However, due to the minimal amount of septal scatter and penetration present with ${ }^{123} \mathrm{I}$ with the ME collimator, the difference in CF values between VOI diameters (ii) and (iii) is less than $2.2 \%$.

The results also show that a less demanding CF source geometry can be used, if appropriate corrections for scatter, attenuation, and CDR (including septal scatter and penetration) are applied during reconstruction. For this reason, the $\mathrm{CF}_{\text {point }}$ was considered optimal for all isotope-collimator combinations, if a VOI diameter equal to the physical size, extended with a radial distance of $3.0 \mathrm{~cm}$ as a margin, is used.

\section{Recovery coefficient curves}


According to literature, the shape of the recovery curves is dependent on several factors, including the size and shape of the object, as well as the presence of activity surrounding the object. (60). In this work, only spherical sources, placed in a water-filled cylindrical phantom, were used to determine the RC curves (Figure 5) for the three isotope-collimator combinations. As a consequence, when applying these RCs as PVE corrections for structures with irregular shapes, the result may be inaccurate (31). It is important to note that the CF source geometry chosen will influence the RCs (27). In this study, the CF was obtained from the $\mathrm{CF}_{\text {point }}$ with a VOI diameter equal to the physical size plus a $3.0 \mathrm{~cm}$ margin. However, the VOI diameter used to determine the recovered activity concentration for the RCs was set equal to the sphere's physical size, since this VOI definition was used to obtain the activity quantification accuracy in the voxel-based digital phantoms. This explains why the RC curves do not reach a value of 1, for all isotope-collimator combinations. The underestimation of the true activity concentration was more pronounced for ${ }^{131} \mathrm{I} \mathrm{HE}$ due to the collimator's limited spatial resolution (60). Similar results have been reported in literature for ${ }^{131}$ I, where different VOI geometries were used to obtain RCs and CFs (61).

\section{Quantification}

Figure 6 showed absolute quantification errors not more than $3.2 \%$ for spheres in an activityfilled cylindrical phantom. The position dependence of VOIs is more pronounced for smaller objects (especially those smaller than two to three times the spatial resolution), which typically results in poorer quantification accuracies. It is important to keep in mind that due to PVEs, smaller objects are generally associated with larger quantification errors (61).

Dewaraja et al. (19) summarised results from physical phantom evaluations of SPECT quantification and highlighted that for ${ }^{131}$ I activity quantification without compensation for PVEs, errors of $17.0 \%$ for large spheres $(8.0 \mathrm{~mL}$ to $95.0 \mathrm{~mL})$, and up to $31.0 \%$ for small spheres $(4.0 \mathrm{~mL})(55)$ were obtained. In another study by Koral et al. (62), ${ }^{131}$ I quantification errors of less than 7.0\% were reported when compensation for PVE was applied. These results highlight the importance of using appropriate PVE corrections to obtain accurate activity quantification. For ${ }^{123}$ I LEHR activity quantification, errors of up to $5.0 \%$ were reported by Shcherbinin et al. (23).

In a recent study, Westerberg (61) reported ${ }^{131}$ I activity quantification errors up to $13.0 \%$ and $8.0 \%$ for small $(10.0 \mathrm{~mL})$ and large $(26.0 \mathrm{~mL})$ spheres, respectively. The author reported an activity quantification error of $35.0 \%$ for the kidney, when no compensation for PVE was 
applied. Brady et al. (12) reported ${ }^{123}$ I activity quantification errors of $10.0 \%$ for spheres with volumes ranging between $5.8 \mathrm{~mL}$ and $29.0 \mathrm{~mL}$.

Our study results showed smaller quantification errors in comparison to results in some of the literature findings, which is most likely attributed to differences in the compensation and reconstruction methods used and the addition of PVE compensation which was often omitted by the referenced authors.

\section{Conclusion}

Our results show when using a simple CF source geometry in clinically realistic patient geometries, it is possible to obtain activity quantification accuracies within $6.0 \%$ for all isotopecollimator combinations, if appropriate corrections for scatter, attenuation, CDR and PVEs were incorporated into the reconstruction of the SPECT data. Our results also show that similar

${ }^{123}$ I activity quantification accuracies could be obtained with the LEHR and ME collimators. For the ${ }^{123}$ I LEHR combination, it requires however that an appropriate correction for septal scatter and penetration are applied. If this is possible, then the ${ }^{123}$ I LEHR combination can be useful for departments that do not have access to a ME collimator. 


$\begin{array}{ll}\text { Abbreviations } \\ { }^{99 \mathrm{~m} T \mathrm{c}} & \text { technetium-99m } \\ { }^{123} \mathrm{I} & \text { iodine-123 } \\ { }^{131} \mathrm{I} & \text { iodine-131 } \\ { }^{177} \mathrm{Lu} & \text { lutetium-177 } \\ \mathrm{CDR} & \text { collimator detector response } \\ \mathrm{CF} & \text { conversion factor } \\ \mathrm{CFs} & \text { conversion factors } \\ \text { HE } & \text { high energy } \\ \text { LEHR } & \text { low energy high resolution } \\ \text { MC } & \text { Monte Carlo } \\ \text { ME } & \text { medium energy } \\ \text { mIBG } & \text { metaiodobenzylguanidine } \\ \text { ML-EM } & \text { maximum likelihood expectation maximisation } \\ \text { NETs } & \text { neuroendocrine tumours } \\ \text { NM } & \text { Nuclear Medicine } \\ \text { OS-EM } & \text { ordered subset expectation maximisation } \\ \text { PVE } & \text { partial volume effect } \\ \text { PVEs } & \text { partial volume effects } \\ \text { RC } & \text { recovery coefficient } \\ \text { RCs } & \text { recovery coefficients } \\ \text { VOIs } & \text { volumes of interest } \\ & \end{array}$

\section{Acknowledgements}

We thank the division of High-Performance Computing at the University of the Free State for the use of the high-performance cluster and their technical support. The authors acknowledge Mrs K Ramonaheng for her editorial assistance.

\section{Code availability}

SIMIND is freely available for download (www.msf.lu.se/research/simind-monte-carloprogram/downloads).

\section{Consent to participate}

Not applicable. 


\section{Authors' contributions}

Conceived and designed the experiments: MM, JvS, HdR and ML. Performed the experiments: MM and JvS. Analysed and interpreted the data: MM, JvS, HdR and ML. Contributed reagents, materials, analysis tools or data: MM, JvS, HdR and ML. Wrote the paper: MM, JvS, HdR and ML. All authors read and approved the final manuscript.

\section{Funding}

This work was funded by the National Research Foundation of South Africa/Swedish Foundation for International Cooperation in Research and Higher Education Science and Technology Research Collaboration, grant (160811184621), as well as by the Medical Research Council of South Africa Flagship Awards Project (SAMRC-RFA-UFSP-012013/HARD), through Dr FCP du Plessis. Partial financial support was received from the Nuclear Technologies in Medicine and Biosciences Initiative (NTeMBI), managed by Necsa.

\section{Availability of data and materials}

The datasets generated during the study are available from the corresponding author on reasonable request.

\section{Ethics approval}

This study was approved by the Health Sciences Research Ethics Committee at the University of the Free State, (UFS-HSD2019/2135/2502). The HSREC functions in compliance with, but not limited to, the following documents and guidelines: The SA National Health Act. No. 61 of 2003; Ethics in Health Research: Principles, Structures and Processes (2015); SA GCP(2006); Declaration of Helsinki; The Belmont Report; The US Office of Human Research Protections 45 CFR 461 (for non-exempt research with human participants conducted or supported by the US Department of Health and Human Services- (HHS), 21 CFR 50, 21 CFR 56; CIOMS; ICH-GCP-E6 Sections 1-4; The International Conference on Harmonization and Technical Requirements for Registration of Pharmaceuticals for Human Use (ICH Tripartite), Guidelines of the SA Medicines Control Council as well as Laws and Regulations with regard to the Control of Medicines, Constitution of the HSREC of the Faculty of Health Sciences.

\section{Consent for publication}

Not applicable.

\section{Competing interests}

The authors declare that they have no competing interests. 


\section{References}

1. Frangos S, Buscombe JR. Why should we be concerned about a "g"? Eur J Nucl Med Mol Imaging. 2019;46:519. doi: 10.1007/s00259-018-4204-z

2. Yordanova A, Eppard E, Kürpig S, Bundschuh RA, Schönberger S, GonzalezCarmona M, et al. Theranostics in nuclear medicine practice. Onco Targets Ther. 2017;10:4821-8. doi:10.2147/ott.s140671

3. Ljungberg M, Gleisner K. Hybrid Imaging for Patient-Specific Dosimetry in Radionuclide Therapy. Diagnostics. 2015;5(3):296-317. doi: 10.3390/diagnostics5030296

4. Wieland DM, Wu J, Brown LE, Mangner TJ, Swanson DP. Radiolabeled Adrenergic Neuron-Blocking Agents : Adrenomedullary Imaging with [ 131I ] Iodobenzylguanidine. J Nucl Med. 1980;21(4):349-53. PMID: 7381563.

5. Sjögreen K, Ljungberg M, Strand S, Library PM. An activity quantification method based on registration of CT and whole-body scintillation camera images, with application to I131. J Nucl Med. 2002;43(7):972-82. doi:10.1.1.580.371

6. Silberstein E. Radioiodine : The Classic Theranostic Agent. Semin Nucl Med. 2012;42(3):164-70. doi: 10.1053/j.semnuclmed.2011.12.002

7. Jimenez C, Erwin W, Chasen B. Targeted radionuclide therapy for patients with metastatic pheochromocytoma and paraganglioma: From low-specific-activity to highspecific-activity iodine-131 metaiodobenzylguanidine. Cancers (Basel). 2019;11(7). doi: 10.3390/cancers11071018

8. Theerakulpisut D, Raruenrom Y, Wongsurawat N, Somboonporn C. Value of SPECT/CT in Diagnostic I-131 MIBG Scintigraphy in Patients with Neuroblastoma. Nucl Med Mol Imaging. 2018;52(5):350-8. doi: 10.1007/s13139-018-0532-y

9. Bombardieri E, Giammarile F, Aktolun C, Baum RP, Bischof Delaloye A, Maffioli L, et al. 131I/123I-Metaiodobenzylguanidine (mIBG) scintigraphy: Procedure guidelines for tumour imaging. Eur J Nucl Med Mol Imaging. 2010;37(12):2436-46. doi: $10.1007 / \mathrm{s} 00259-003-1357-0$

10. Giammarile F, Chiti A, Lassmann M, Brans B, Flux G. EANM procedure guidelines 
for 131I-meta-iodobenzylguanidine (131I-mIBG) therapy. Eur J Nucl Med Mol Imaging. 2008;35(5):1039-47. doi: 10.1007/s00259-008-0715-3

11. Dewaraja YK, Ljungberg M, Green AJ, Zanzonico PB, Frey EC. MIRD Pamphlet No. 24: Guidelines for Quantitative 131 I SPECT in Dosimetry Applications. J Nucl Med. 2013;54(12):2182-9. doi: 10.2967/jnumed.113.122390

12. Brady SL, Shulkin BL. Analysis of quantitative [ I-123 ] mIBG SPECT / CT in a phantom and in patients with neuroblastoma. EJNMMI Phys. 2019;6(31). doi: 10.1186/s40658-019-0267-6

13. Flotats A, Carrio I. I-mIBG and the phantom tollbooth. J Nucl Cardiol. 2017;25(3):1198-200. doi: 10.1007/s12350-017-0843-х

14. Inoue Y, Shirouzu I, Machida T, Yoshizawa Y, Akita F, Doi I, et al. Physical characteristics of low and medium energy collimators for 123 I imaging and simultaneous dual-isotope imaging. Nucl Med Commun. 2003;24:1195-202. doi: 10.1097/00006231-200311000-00011

15. Dobbeleir AA, Hambÿe AE, Franken PR. Influence of high-energy photons on the spectrum of iodine-123 with low- and medium-energy collimators : consequences for imaging with 123 I-labelled compounds in clinical practice. Eur J Nucl Med. 1999;26(6):655-8. doi: 10.1007/s002590050434

16. Rault E, Vandenberghe S, Holen R Van, Beenhouwer J De, Staelens S, Lemahieu I. Comparison of image quality of different iodine isotopes ( I-123, I-124, and I-131 ). Cancer Biother Radiopharm. 2007;22(3). doi: 10.1089/cbr.2006.323

17. Shepp LA, Vardi Y. Maximum likelihood reconstruction for emission tomography. IEEE Trans Med Imaging. 1982;1:113-22. doi: 10.1109/TMI.1982.4307558

18. Lange K, Carson R. EM Reconstruction Algorithms for Emission and Transmission Tomography. J Comput Assist Tomogr. 1984;8(2):306-16. doi: PMID: 6608535.

19. Dewaraja YK, Frey EC, Sgouros G, Brill AB, Roberson P, Zanzonico PB, et al. MIRD pamphlet no. 23: Quantitative SPECT for patient-specific 3-dimensional dosimetry in internal radionuclide therapy. J Nucl Med. 2012;53(8):17. doi:

10.2967/jnumed.111.100123 
20. D'Arienzo M, Cazzato M, Cozzella ML, Cox M, D'Andrea M, Fazio A, et al. Gamma camera calibration and validation for quantitative SPECT imaging with 177Lu. Appl Radiat Isot. 2016;112:156-64. doi: 10.1016/j.apradiso.2016.03.007

21. Zhao W, Esquinas PL, Hou X, Uribe CF, Gonzalez M, Beauregard J, et al. Determination of gamma camera calibration factors for quantitation of therapeutic radioisotopes. EJNMMI Phys. 2018;5(8):16. doi: 10.1186/s40658-018-0208-9

22. Ljungberg M, Sjögreen Gleisner K. Personalized Dosimetry for Radionuclide Therapy Using Molecular Imaging Tools. Biomedicines. 2016;4(4):25. doi:

10.3390/biomedicines4040025

23. Shcherbinin S, Celler A, Belhocine T, Vanderwerf R, Driedger A. Accuracy of quantitative reconstructions in SPECT / CT imaging. Phys Med Biol. 2008;53:4595604. doi: 10.1088/0031-9155/53/17/009

24. Willowson K, Bailey D, Baldock C. Quantitative SPECT reconstruction using CTderived corrections. Phys Med Biol. 2008;53(12):3099-112. doi: 10.1088/0031$9155 / 53 / 12 / 002$

25. Cherry SR, Sorenson J, Phelps ME, Methé BM. Physics in Nuclear Medicine. 4th ed. Philadelphia: Saunders; 2012. 1-544 p.

26. Hoffman E, Huang S, Phelps M. Quantitation in positron emission computed tomography: 1. Effect of object size. J Comput Assist Tomogr. 1979;3(3):299-308. doi: 10.1097/00004728-197906000-00001

27. Ramonaheng K, van Staden JA, du Raan H. The Effect of Calibration Factors and Recovery Coefficients on 177Lu SPECT Activity Quantification Accuracy: A Monte Carlo study. EJNMMI Phys. 2021;8:27. doi: 10.1186/s40658-021-00365-8

28. Ritt P, Vija H, Hornegger J, Kuwert T. Absolute quantification in SPECT. Eur J Nucl Med Mol Imaging. 2011;38:69-77. doi: 10.1007/s00259-011-1770-8

29. Zeintl J, Vija AH, Yahil A, Hornegger J, Kuwert T. Quantitative Accuracy of Clinical 99mTc SPECT/CT Using Ordered-Subset Expectation Maximization with 3Dimensional Resolution Recovery, Attenuation, and Scatter Correction. J Nucl Med. 2010;51(6):921-8. doi: 10.2967/jnumed.109.071571 
30. Dewaraja YK, Ljungberg M, Koral KF. Monte Carlo evaluation of object shape effects in iodine-131 SPET tumor activity quantification. Eur J Nucl Med. 2001;28(7):900-6. doi: $10.1007 / \mathrm{s} 002590100551$

31. Tran-Gia J, Lassmann M. Optimizing image quantification for Lu-177 SPECT/CT based on a 3D printed 2-compartment kidney phantom. J Nucl Med. 2018;59:616-24. doi: 10.2967/jnumed.117.200170

32. Bahreyni Toossi M., Islamian P, Momennezhad M, Ljungberg M, Naseri S. SIMIND Monte Carlo simulation of a single photon emission CT. J Med Phys. 2010;35(1):42. doi: 10.4103/0971-6203.55967

33. Ljungberg M, Celler A, Konijnenberg MW, Eckerman KF, Dewaraja YK, SjögreenGleisner K. MIRD pamphlet no. 26: Joint EANM/MIRD guidelines for quantitative 177Lu SPECT applied for dosimetry of radiopharmaceutical therapy. J Nucl Med. 2016;57(1):151-62. doi: 10.2967/jnumed.115.159012

34. Ljungberg M, Strand S-E, King M a. The SIMIND Monte Carlo program. Monte Carlo Calc Nucl Med Appl diagnostic imaging. 1998;145-63. https://lup.lub.lu.se/record/3217210

35. Zaidi H. Relevance of accurate Monte Carlo modeling in nuclear medical imaging. Med Phys. 1999;26(February):574-608. doi: 10.1118/1.598559

36. Fahey FH, Grogg K, El Fakhri G. Use of Monte Carlo Techniques in Nuclear Medicine. J Am Coll Radiol. 2018;15(3):446-8. doi: 10.1016/j.jacr.2017.09.045

37. Autret D, Bitar A, Ferrer L, Lisbona A, Bardiès M. Monte Carlo modeling of gamma cameras for I-131 imaging in targeted radiotherapy. Cancer Biother Radiopharm. 2005;20(1):77-84. doi: 10.1089/cbr.2005.20.77

38. Ljungberg M. Absolute Quantitation of SPECT Studies. Semin Nucl Med. 2018;48(4):348-58. doi: 10.1053/j.semnuclmed.2018.02.009

39. Ljungberg M, Strand S. A Monte Carlo program for the simulation of scintillation camera characteristics. Comput Methods Programs Biomed. 1989;29(4):257-72. doi: 10.1016/0169-2607(89)90111-9

40. Knoll GF. Radiation Detection and Measurement. Vol. 3 edition, John Wiley \& Sons. 
Inc. $2005.816 \mathrm{p}$.

41. Morphis M, van Staden JA, du Raan H, Ljungberg M. Modelling of energy-dependent spectral resolution for SPECT Monte Carlo simulations using SIMIND. Heliyon. 2021;7:12. doi: 10.1016/j.heliyon.2021.e06097

42. Ejeh J. Accuracy of iodine-131 activity quantification and dosimetry for threedimensional patient-specific models. 2019. [thesis] University of the Free State, South Africa

43. Dewaraja YK, Wilderman SJ, Ljungberg M, Koral KF, Zasadny K, Kaminiski MS. Accurate dosimetry in 131I radionuclide therapy using patient-specific, 3-dimensional methods for SPECT reconstruction and absorbed dose calculation. J Nucl Med. 2005;46(5):840-9. PMID: 15872359

44. Morphis M, van Staden JA, du Raan H, Ljungberg M. Validation of a SIMIND Monte Carlo modelled gamma camera for Iodine-123 and Iodine-131 imaging. Heliyon. 2021; [in press].

45. Fluke Biomedical. Nuclear Associates 76-823, 76-824 \& 76-825 PET/SPECT Phantom Source Tank, Phatom Inserts and Cardiac Insert User Guide. 2005. p. 10.

46. Ramonaheng K, van Staden J, du Raan H. Validation of a Monte Carlo Modelled Gamma Camera for Lutetium-177 Imaging. Appl Radiat Isot. 2020;163. doi: 10.1016/j.apradiso.2020.109200

47. Sjögreen K, Ljungberg M, Wingårdh K, Minarik D, Strand S-E. The LundADose method for planar image activity quantification and absorbed-dose assessment in radionuclide therapy. Cancer Biother Radiopharm. 2005;20(1):92-7. doi: $10.1089 /$ cbr.2005.20.92

48. Frey EC, Tsui BMW. A new method for modeling the spatially-variant, objectdependent scatter response function in SPECT. In: 1996 IEEE Nuclear Science Symposium Conference Record. IEEE; 1996. p. 1082-6. doi: 10.1109/NSSMIC.1996.591559

49. Ljungberg M, Sjogreen K, Liu X, Frey E, Dewaraja Y, Strand S-E. A 3-Dimensional Absorbed Dose Calculation Method Based on Quantitative SPECT for Radionuclide 
Therapy : Evaluation for 131 I Using Monte Carlo Simulation. J Nucl Med. 2002;43:1101-9. PMID: 12163637

50. Loening AM, Gambhir SS. AMIDE: A Completely Free System for Medical Imaging Data Analysis. J Nucl Med. 2001;42(5):192. doi: 10.1.1.532.9777

51. Brambilla M, Cannillo B, Dominietto M, Inglese E. Characterization of orderedsubsets expectation maximization with 3D post- reconstruction Gauss filtering and comparison with filtered backprojection. Ann Nucl Med. 2005;19(2):75-82. doi: 10.1007/BF03027384

52. Leong LK, Kruger RL, Connor MKO. A Comparison of the Uniformity Requirements for SPECT Image Reconstruction Using FBP and OSEM Techniques. J Nucl Med Technol. 2001;29:79-83. PMID: 11376099

53. Sjögreen K, Ljungberg M, Strand S. Parameters Influencing Volume and Activity Quantitation in Spect. Acta Oncol (Madr). 1996;35(4):323-30. doi: $10.3109 / 02841869609101649$

54. Nakajima K, Verschure DO, Okuda K, Verberne HJ. Standardization of 123 I- meta iodobenzylguanidine myocardial sympathetic activity imaging : phantom calibration and clinical applications. Clin Transl Imaging. 2017;5(3):255-63. doi: $10.1007 / \mathrm{s} 40336-017-0230-2$

55. Dewaraja YK, Koral KF, Fessler JA. Regularized reconstruction in quantitative SPECT using CT side information from hybrid imaging. Phys Med Biol. 2010;55:2523-39. doi: 10.1088/0031-9155/55/9/007

56. Koral KF, Kritzmaan JN, Rogers VE, Ackermann RJ, Fessler JA. Optimizing the number of equivalent iterations of 3D OSEM in SPECT reconstruction of I-131 focal activities. Nucl Instruments Methods Phys Res. 2007;579(A):326-9. doi: 10.1016/j.nima.2007.04.070

57. Grassi E, Mezzenga E, Finocchiaro D. Impact of a commercial 3D OSEM reconstruction algorithm on the $177 \mathrm{Lu}$ activity quantification of SPECT / CT imaging in a Molecular Radiotherapy trial. Radiol Diagnostic Imaging. 2017;1(1):1-7. doi: 10.15761/RDI.1000101 
58. Tran-gia J, Lassmann M. Characterization of Noise and Resolution for Quantitative 177 Lu SPECT/CT with xSPECT Quant. J Nucl Med. 2019;60(1):50-9. doi: 10.2967/jnumed.118.211094

59. Niñerola-Baizán A, Gallego J, Cot A, Aguiar P, Lomeña F, Pavía J, et al. Optimization of the reconstruction parameters in [ 123 I ] FP-CIT. Phys Med Biol. 2018;63:10. doi: 10.1088/1361-6560/aab799

60. Frey EC, Humm JL, Ljungberg M. Accuracy and precision of radioactivity quantification in nuclear medicine images. Semin Nucl Med . 2012;42(3):208-18. doi: 10.1053/j.semnuclmed.2011.11.003

61. Westerberg F, Hindorf C, Larsson E, Stenvall A. Quantification of I-131 Activity from Gamma Camera Images of Thyroid Cancer Patients. 2019. [thesis] Lund University, Lund, Sweden

62. Koral KF, Yendiki A, Lin Q, Dewaraja YK. Comparison of 3-D OSEM Versus 1-D SAGE for Focal Total-Activity Quantification in I-131 SPECT With HE Collimation. IEEE Trans Nucl Sci. 2005;52(1):154-8. doi: 10.1109/tns.2004.843154 


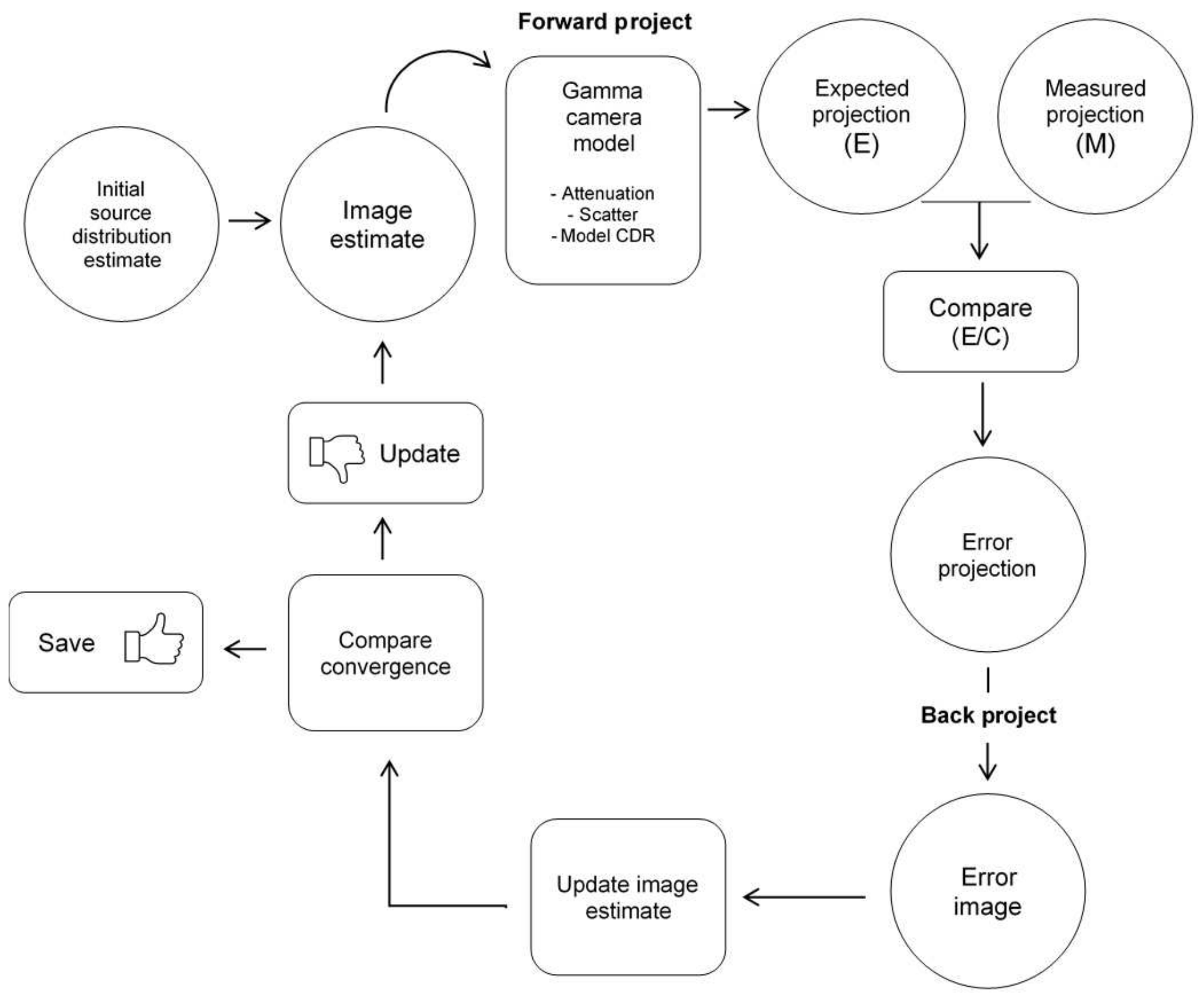

Figure 1

Schematic describing basic steps of iterative reconstruction method. 
(a)

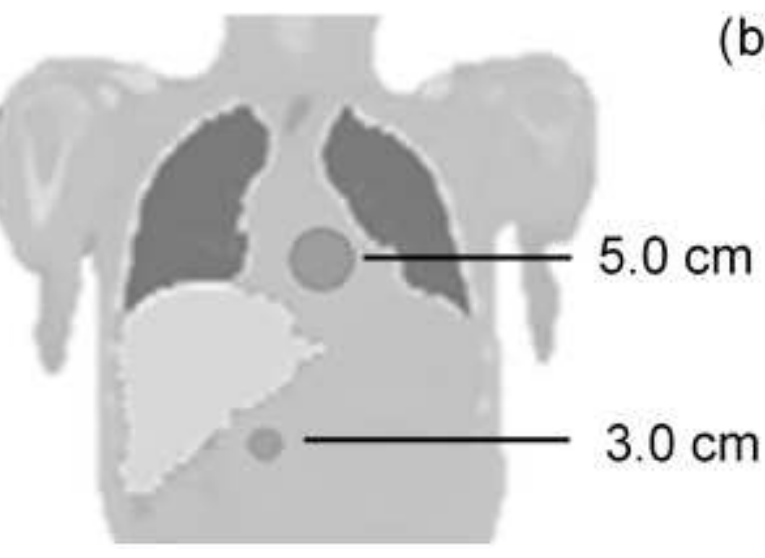

(b)

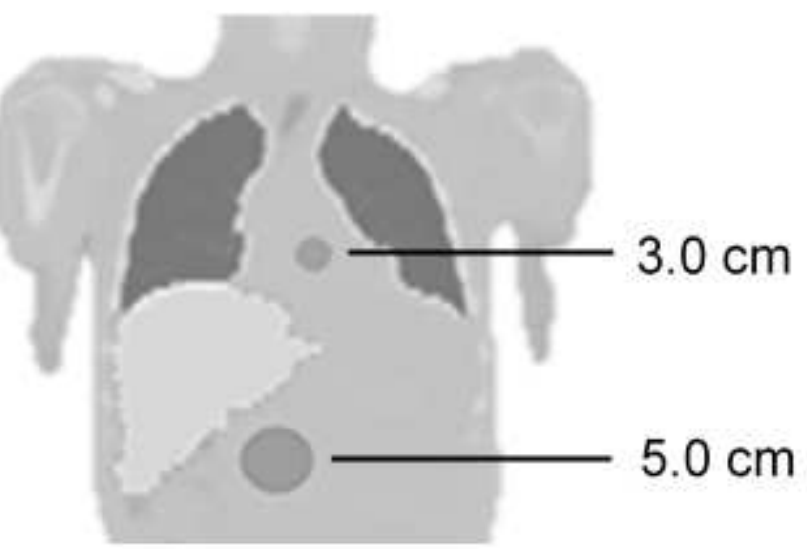

Figure 2

Segmented coronal CT slices of the voxel-based digital patient phantom with spheres' positions for (a) scenario 1 and (b) scenario 2. 
(a) ${ }^{123}$ LEHR

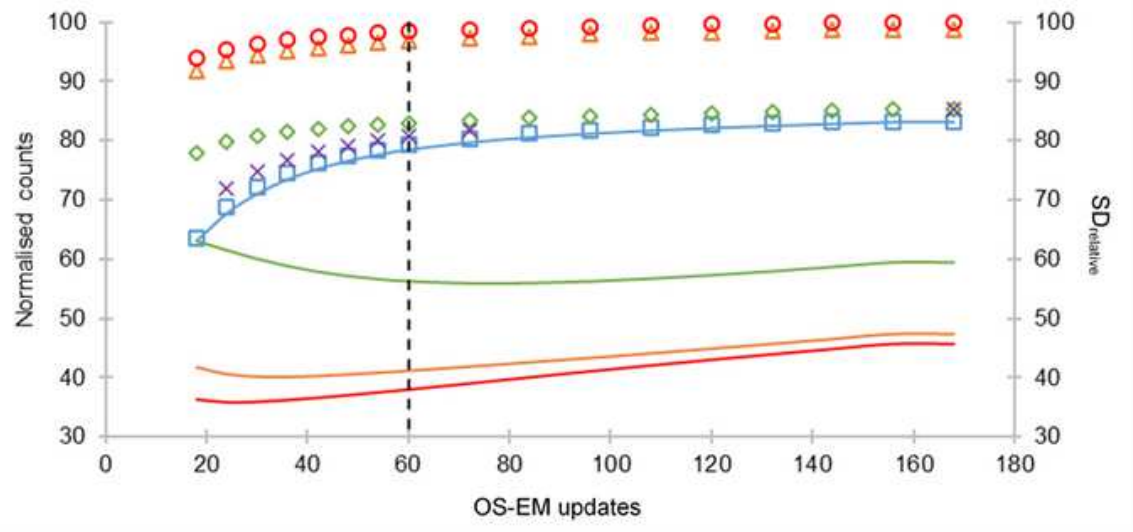

(b) ${ }^{123} \mathrm{ME}$

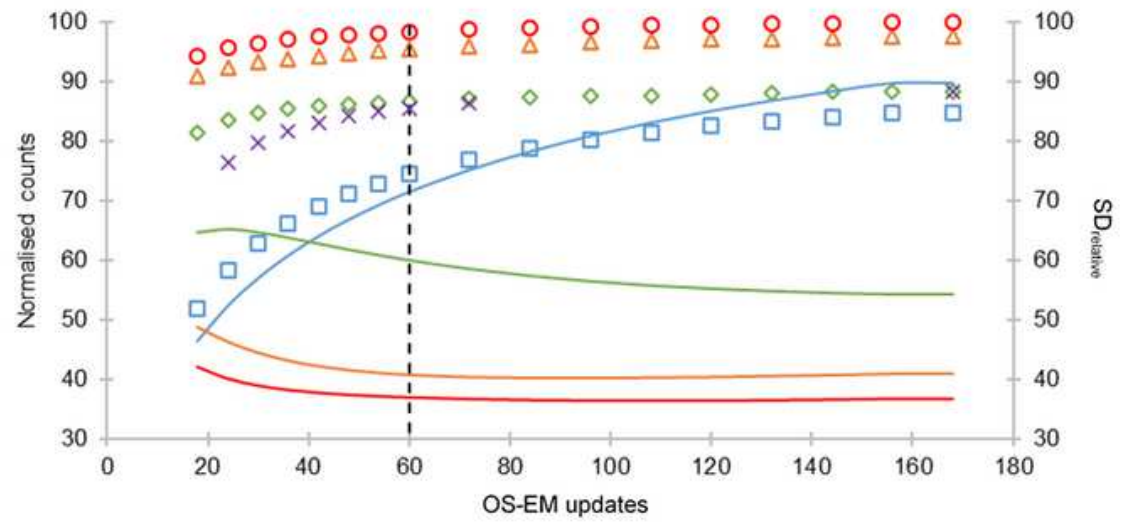

(c) ${ }^{131} \mathrm{I} \mathrm{HE}$

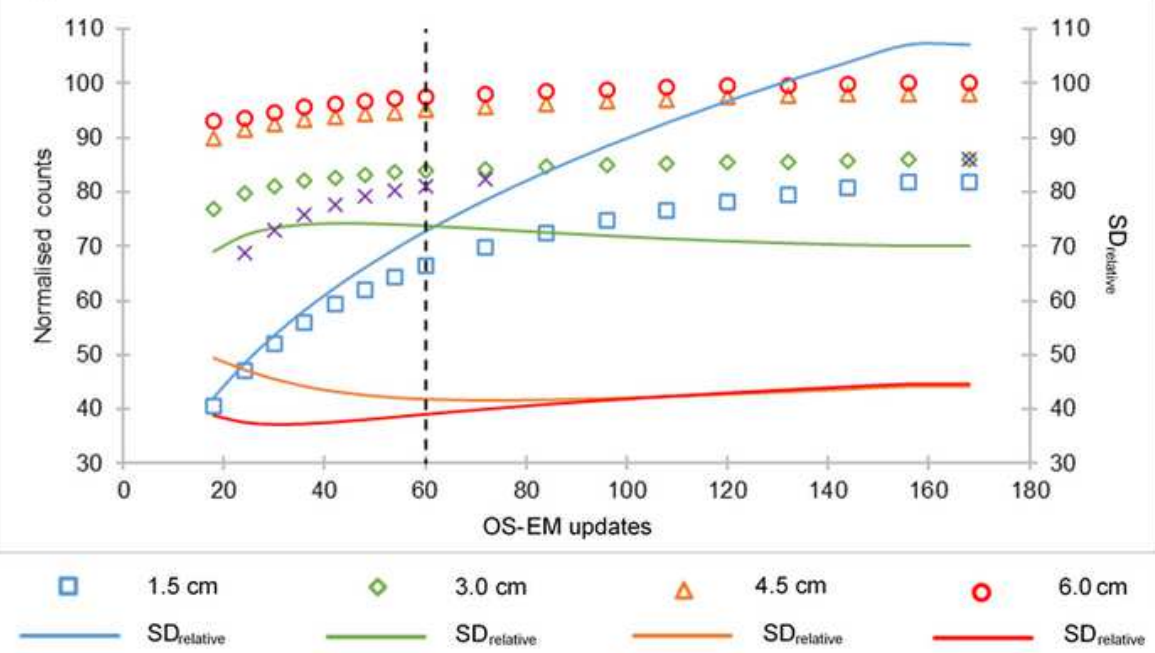

- - - Optimal OS-EM updates $\quad \times \quad$ Voxel-based digital patient phantom $3.0 \mathrm{~cm}$

\section{Figure 3}

Normalised counts and SD_relative as a function of OS-EM updates for spheres with diameters of $1.5 \mathrm{~cm}$, $3.0 \mathrm{~cm}, 4.5 \mathrm{~cm}$, and $6.0 \mathrm{~cm}$, for (a) 123I LEHR, (b) $123 \mathrm{I} \mathrm{ME}$ and (c) $131 \mathrm{I} \mathrm{HE}$. 

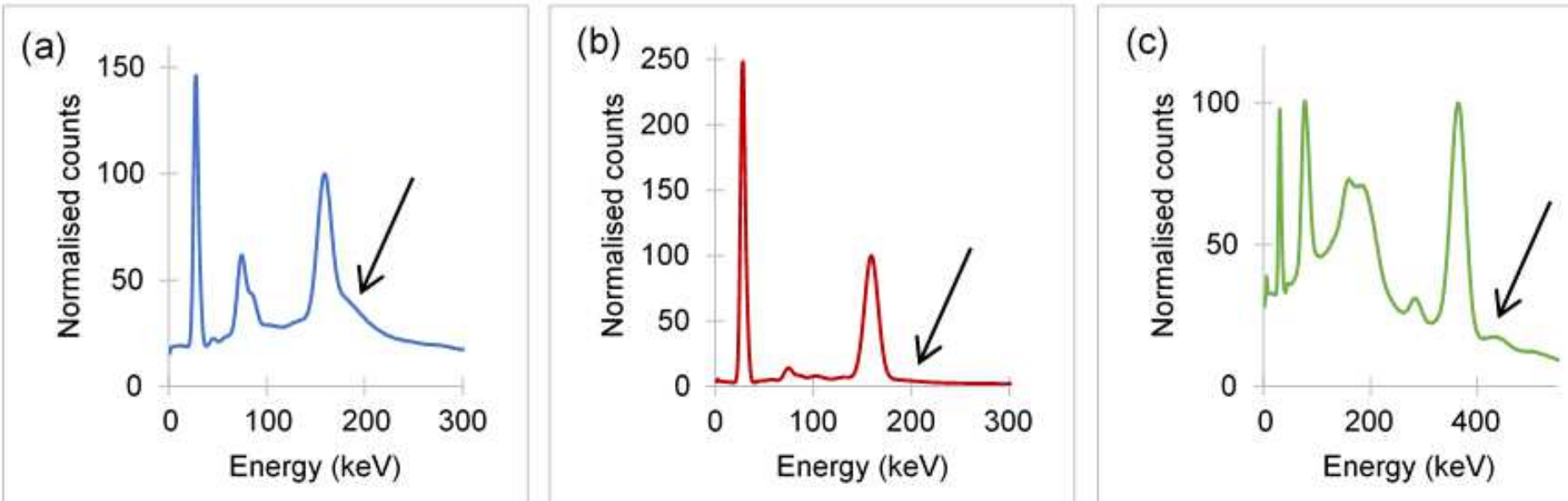

\section{Figure 4}

Simulated energy spectra of CFpoint projection images for (a) 123I LEHR, (b) 123I ME, and (c) 131I HE.

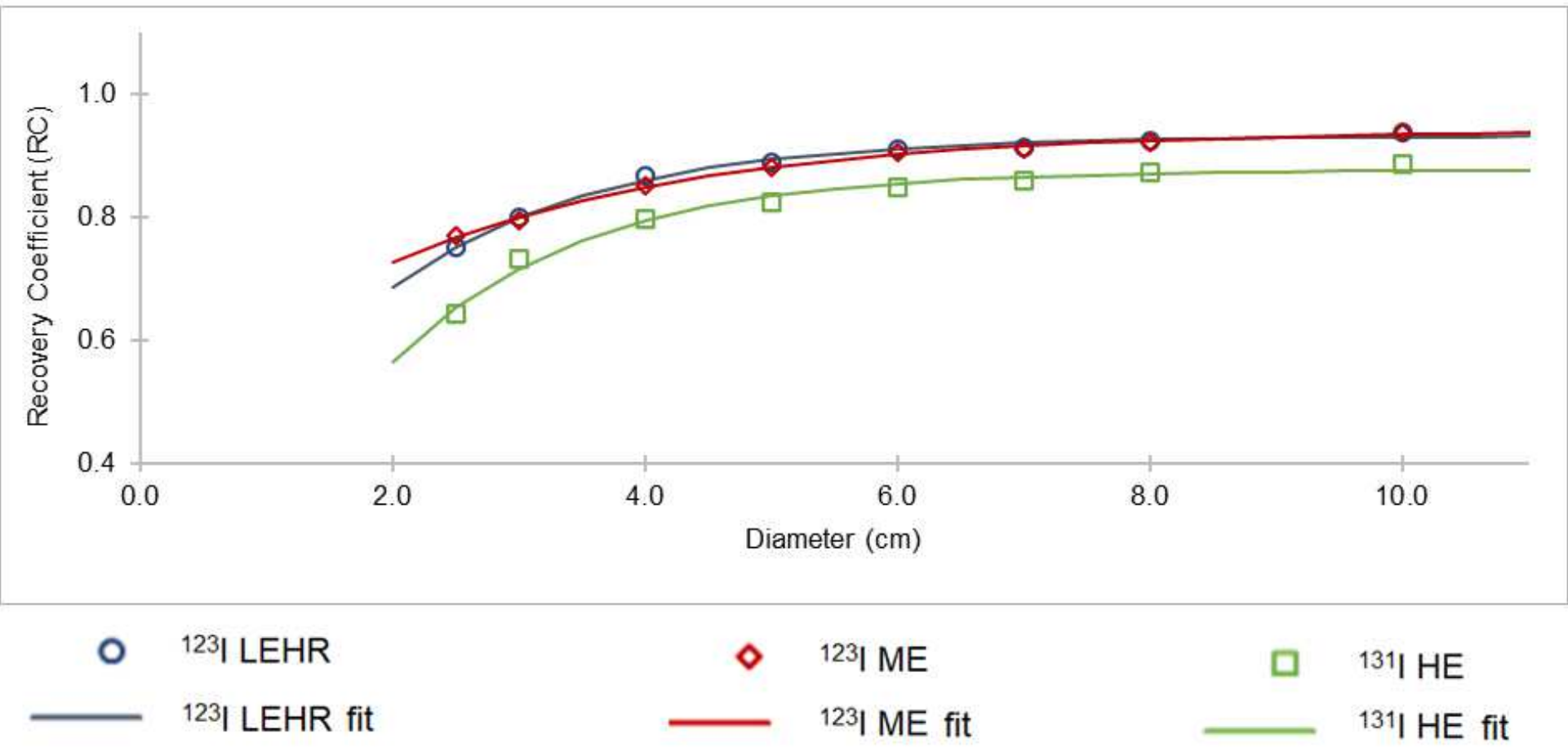

Figure 5

$\mathrm{RC}$ as a function of sphere diameter for 123I LEHR, 123I ME and 131I HE, with the solid lines showing the fitted functions. 


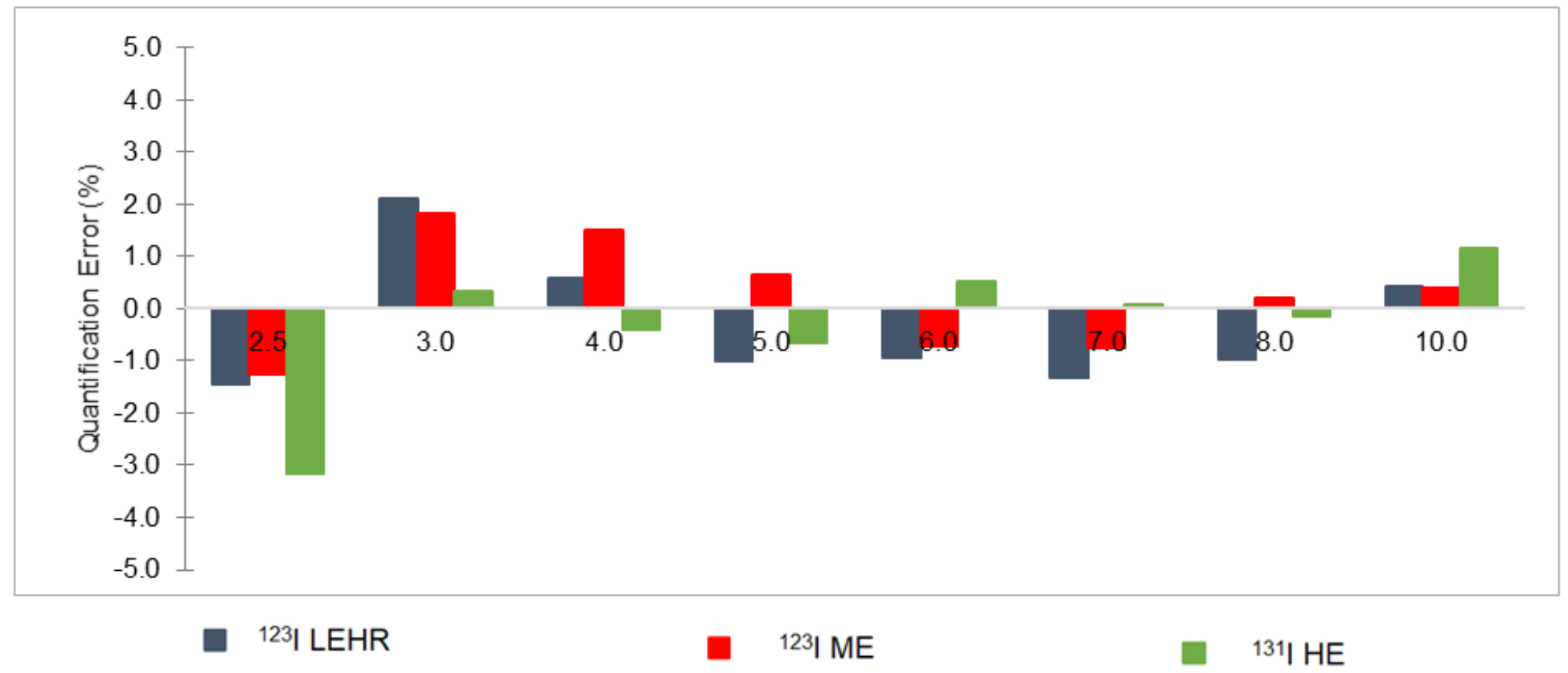

Figure 6

Quantification error (\%) of spheres of differing diameter in a warm cylindrical phantom, for 123I LEHR, 123I ME and 131I HE. 


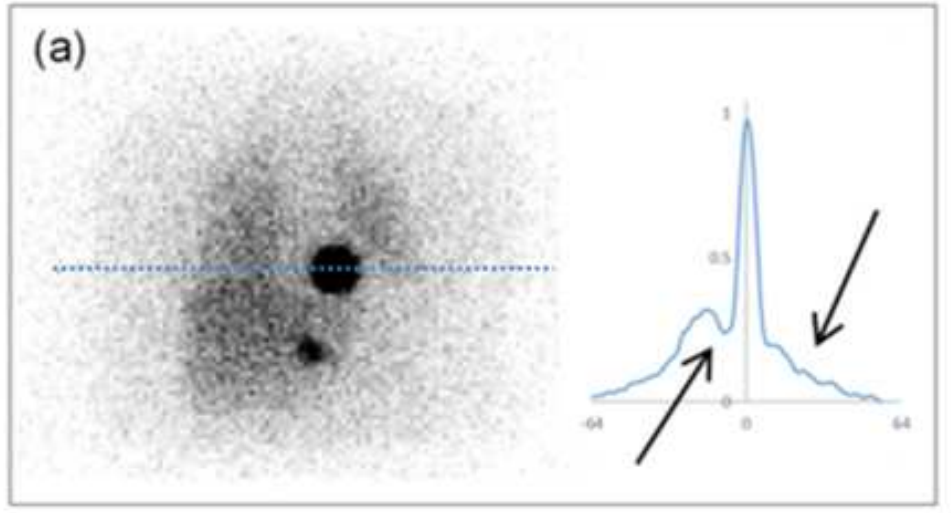

(b)

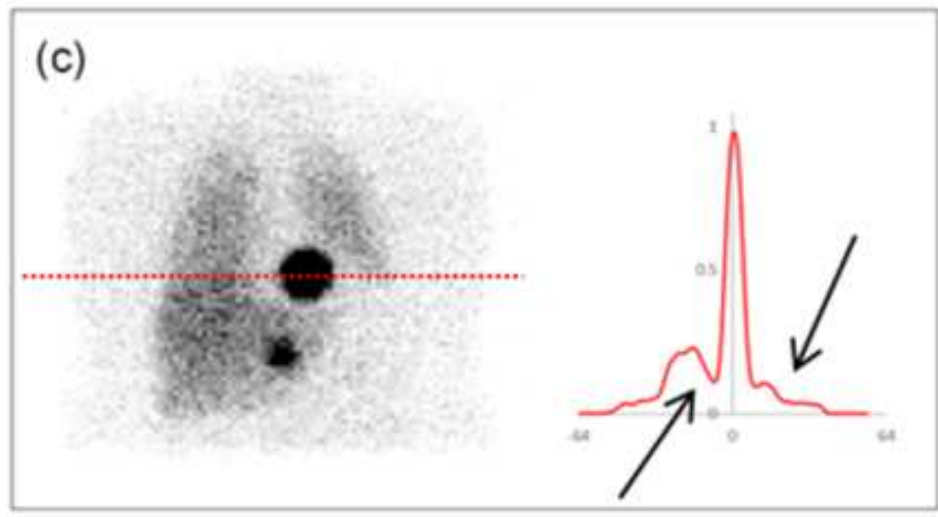

(d)
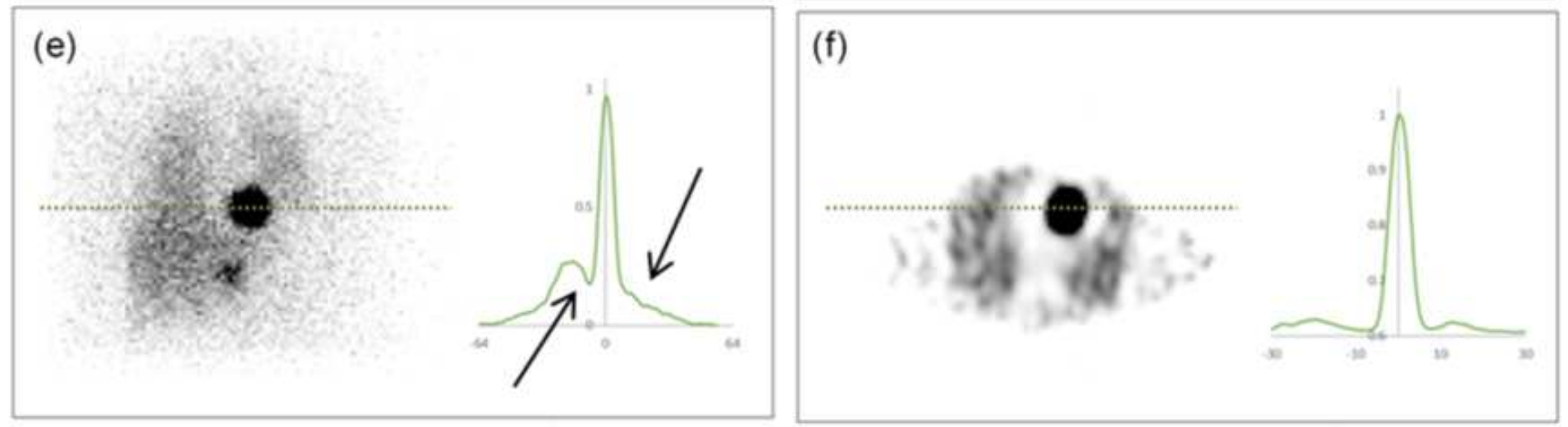

Figure 7

Uncorrected projection images (a, c, e), and reconstructed corrected transaxial slices $(b, d, f)$ through the voxel-based digital patient phantom for $(a, b) 123$ I LEHR, (c, d) 123I ME, and (e, f) 131I HE. Profiles through the largest sphere are displayed alongside their respective images. Arrows indicate septal scatter and penetration at peak and trough and at tail region. 


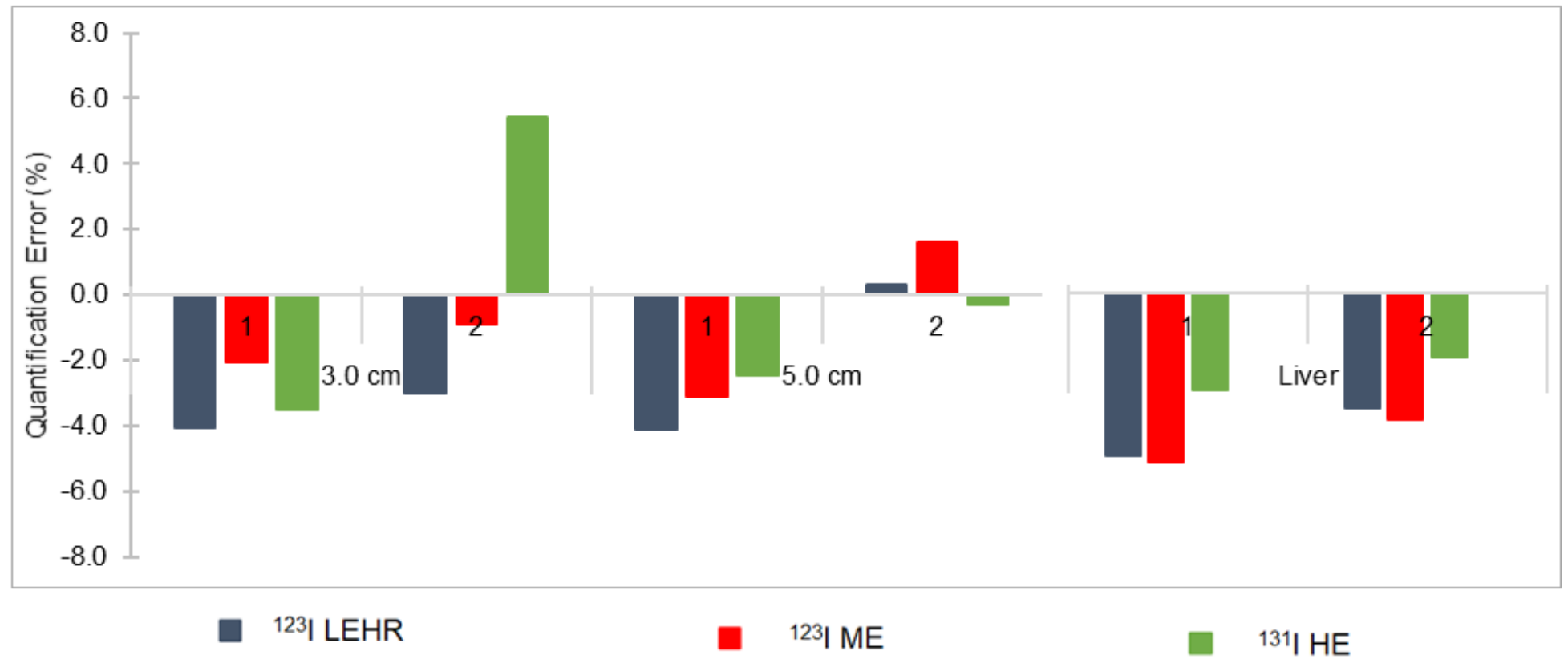

Figure 8

Activity quantification errors (\%) for spheres in the voxel-based digital patient phantom (scenario 1 and 2), for 123I LEHR, 123I ME, and 131I HE. 